Journal for ImmunoTherapy of Cancer

\section{LINC01140 promotes the progression and tumor immune escape in lung cancer by sponging multiple microRNAs}

To cite: Xia R, Geng G, Yu X, et al. LINC01140 promotes the progression and tumor immune escape in lung cancer by sponging multiple microRNAs. Journal for ImmunoTherapy of Cancer 2021;9:e002746. doi:10.1136/jitc-2021-002746

- Additional supplemental material is published online only. To view, please visit the journal online (http://dx.doi.org/10. 1136/jitc-2021-002746).

RX, GG, XY and ZX contributed equally.

Accepted 26 July 2021

Check for updates

(C) Author(s) (or their employer(s)) 2021. Re-use permitted under CC BY-NC. No commercial re-use. See rights and permissions. Published by BMJ.

For numbered affiliations see end of article.

Correspondence to Professor Yanjun Mi; Miyj77@xmu.edu.cn

Professor Jie Jiang; Jiejiangfy@163.com

Professor Qicong Luo; rokey526@163.com

Professor Xiaofang Dai; 13707127709@163.com

\section{ABSTRACT}

Background Long intergenic non-protein coding RNA 1140 (LINC01140), a long non-coding RNA, is highly expressed in various cancers; however, its biological functions in lung cancer (LC) progression and immune escape are still unclear.

Methods Here, to elucidate LINC01140 function, 79 paired LC and paracancerous tissues were collected. LINC01140 expression levels were determined using fluorescence in situ hybridization and qPCR analysis. Cell counting kit-8 (CCK-8) assay and transwell assays were performed. The interaction between microRNAs (miRNAs) and LINC01140 was confirmed using an RNA immunoprecipitation assay. Cytokine-induced killer (CIK) cell phenotypes were analyzed by flow cytometry. Cytokine secretion levels were determined by ELISA. CIK cytotoxicity was assessed by measuring lactate dehydrogenase release. Besides, xenograft tumor mouse models were used to unveil the in vivo function of LINC01140.

Results We found that LINC01140 was highly expressed in human LC tissues and cell lines. High LINC01140 levels were associated with poor survival in patients with LC. LINC01140 upregulation promoted the proliferation, migration, and invasion of LC cells through direct interaction with miR-33a-5p and miR-33b-5p, thereby contributing to $\mathrm{c}-\mathrm{Myc}$ expression and also inhibited cisplatin-induced cell apoptosis. In subcutaneous tumor xenograft mice, LINC01140 knockdown markedly reduced tumor growth and lung metastasis. Additionally, LINC01140 directly repressed miR-377-3p and miR-155$5 p$ expression levels, resulting in the upregulation of their common downstream target programmed death-ligand 1 (PD-L1), a crucial target in LC immunotherapy. Notably, we proved that LINC01140 knockdown, along with CIK administration, suppressed the growth of subcutaneous LC xenografts by decreasing PD-L1 expression in severe combined immunodeficient mice.

Conclusions Taken together, LINC01140 overexpression protects c-Myc and PD-L1 mRNA from miRNA-mediated inhibition and contributes to the proliferation, migration, invasion, and immune escape of LC cells. These results provide a theoretical basis that LINC01140 is a promising target for LC treatment.

\section{BACKGROUND}

Lung cancer (LC) is the leading cause of cancer-related morbidity and mortality worldwide, resulting in millions of deaths annually. ${ }^{1}$ The 5-year survival rate for LC remains poor. $^{2}$ Histologically, patients with non-small cell LC (NSCLC) accounts for approximately $85 \%$ of all LC cases. ${ }^{3}$ At early stage, surgical resection contributes to a favorable outcome; however, as symptoms of early cancer are difficult to identified, most patients are diagnosed at an advanced or metastatic stage ${ }^{45}$ and could hardly benefit from systemic chemotherapy. ${ }^{6}$ This highlights the need to identify novel biomarker to improve the early diagnosis of LC.

Most of the human genome is transcribed into non-coding RNA, most of which functions in the regulation of gene expression. ${ }^{7}$ Long non-coding RNAs (lncRNAs) have been identified as key regulators of numerous biological processes, including development, differentiation, cell fate determination, and disease pathogenesis. ${ }^{8-12}$ LncRNAs exert their biological functions by acting as signaling mediators, molecular decoys, scaffolds, or transcriptional enhancers. ${ }^{13}$ Interestingly, many lncRNAs serve as competitive endogenous RNAs that regulate gene expression by sponging microRNAs (miRNAs). For instance, high expression of the lncRNA SNHG1 in LC tissues promotes the progression of NSCLC by reducing miR-101-3 p level and activating the Wnt/ $\beta$-catenin signaling pathway. ${ }^{14}$ Caveolin-1 enhances the proliferation and metastatic potential of LC cells by upregulating the expression of lncRNA HOX transcript antisense RNA (HOTAIR). ${ }^{15}$ However, the role played by many lncRNAs in LC development remains unclear.

LncRNAs are classified into various types, including long intergenic non-coding RNAs (lincRNAs) and antisense lncRNAs. ${ }^{16}$ Here, we focused on long intergenic non-protein coding RNA 1140 (LINC01140), which is reported to be upregulated in gastric cancer, while LINC01140 along with two other 
lncRNAs can serve as a marker of the clinical prognosis of gastric cancer. ${ }^{17}$ Additionally, LINC01140 is markedly upregulated during pulmonary fibrosis and modulates the inflammatory response of the lung, further promoting fibrotic lesions. LINC01140 knockdown helped to reduce the secretion of inflammatory cytokine factors into the microenvironment and to alleviate pulmonary fibrosis progression. ${ }^{18}$

Here, we initially confirmed that LINC01140 was highly expressed in LC tissues and then showed that LINC01140 promoted the proliferation, migration, invasion, and immune escape of LC cells by adsorbing several miRNAs known to function as tumor suppressors. Our findings provide new insights into LC development and identify a potential target for LC treatment.

\section{METHODS}

\section{Human LC tissues}

A total of 79 paired primary LC tumor tissues and adjacent normal lung tissues were obtained from the First Affiliated Hospital of Xiamen University between 2015 and 2019. None of the patients had received preoperative chemotherapy or radiation therapy or adjuvant therapies. One part of each sample was formalin fixed and paraffin embedded, while the other was stored in liquid nitrogen for subsequent experiments. Clinicopathological characteristics of the 79 patients with LC are listed in table 1.

\section{Cell culture}

Human NSCLC cells A549, 95-D, NCI-H226, and H1975, human mucoepidermoid bronchiolar carcinoma cell line NCI-H292 (hereafter H292), human lung squamous carcinoma cells EBC-1, normal human lung epithelial cell line BEAS-2B, human bronchial epithelial (HBE) cells, and 293T cells were purchased from American Type Culture Collection (Manassas, Virginia, USA). LC cells were grown in RPMI1640 medium (Gibco) containing $10 \%$ fetal bovine serum (Gibco) and $1 \%$ penicillin-streptomycin (Gibco). BEAS-2B, ${ }^{19}{ }^{20} \mathrm{HBE}$, and 293T cells were cultured using Dulbecco's modified Eagle's medium (DMEM) medium (Gibco) plus 10\% fetal bovine serum and $1 \%$ penicillin-streptomycin. All cells were kept at $37^{\circ} \mathrm{C}$ in a humidified culture incubator gassed with $5 \%$ $\mathrm{CO}_{2}$.

\section{Cytokine-induced killer (CIK) cell preparation}

Peripheral blood was collected from patients into vacutainer tubes containing heparin (anticoagulant). Peripheral blood mononuclear cells (PBMCs) were isolated by Ficoll-Conray density gradient centrifugation, rinsed three times with phosphate-buffered saline (PBS, $\mathrm{pH} 7.4)$, and seeded into six-well plates $\left(2 \times 10^{6}\right.$ cells $\left./ \mathrm{mL}\right)$. The cells were then cultured in RPMI 1640 supplemented with $10 \%$ inactivated human serum and maintained at $37^{\circ} \mathrm{C}$ in a saturated atmosphere containing $5 \% \mathrm{CO}_{2}$.

On day 1 , recombinant human interferon-gamma $\left(\mathrm{IFN}-\gamma, \quad 1 \times 10^{6} \mathrm{U} / \mathrm{L}\right)$, recombinant human interleukin
Table 1 The clinicopathological factors of patients (79 cases)

\begin{tabular}{ll}
\hline Characteristics & Number of cases (\%) \\
\hline $\begin{array}{l}\text { Age (years) } \\
\leq 60\end{array}$ & $47(59.5)$ \\
\hline$>60$ & $32(40.5)$ \\
\hline $\begin{array}{l}\text { Gender } \\
\text { Male }\end{array}$ & \\
\hline Female & $43(54.4)$ \\
\hline Smoking & $36(45.6)$ \\
\hline Yes & \\
\hline No & $57(72.2)$ \\
\hline Pathological types & $22(27.8)$ \\
\hline Adenocarcinoma & \\
\hline Squamous carcinoma & $41(51.9)$ \\
\hline Differentiation degree & $38(48.1)$ \\
\hline High & \\
\hline Moderate & $19(24.1)$ \\
\hline Low & $34(43)$ \\
\hline TNM stage & $26(32.9)$ \\
\hline I+II & \\
\hline III & $58(73.4)$ \\
\hline Tumor size (cm) & $21(26.6)$ \\
\hline$<3$ & \\
\hline$\geq 3$ & $59(74.7)$ \\
\hline Lymph node metastasis & $20(25.3)$ \\
\hline No & \\
\hline Yes & $44(55.7)$ \\
\hline Distant metastasis & $35(44.3)$ \\
\hline No & $61(77.2)$ \\
\hline Yes & $18(22.8)$ \\
\hline TNM tumor-nos & \\
\hline
\end{tabular}

TNM, tumor-node-metastasis.

(IL) - $2\left(5 \times 10^{5} \mathrm{U} / \mathrm{L}\right), 25 \mathrm{M}$ HEPES, and $2 \mathrm{mM} \mathrm{L-glu-}$ tamine were added into the culture medium. Twentyfour hours later, monoclonal antibodies targeting human CD3 $(100 \mu \mathrm{g} / \mathrm{L}$, Antibody Diagnostic, New York, USA) and IL-1 $\alpha\left(1 \times 10^{5} \mathrm{U} / \mathrm{L}\right)$ were added. After 2 days of culture, culture medium was replaced with IFN- $\gamma$-free medium and changed every 3 days. CIK cells were harvested 12-14 days and the percentage of live cells was calculated using trypan blue $(0.2 \%)$ staining.

\section{Xenograft mouse models}

The animal experiments were approved by the Animal Care and Use Committee of the First Affiliated Hospital of Xiamen University. Eight-week-old female severe combined immunodeficient mice, weighing between $20 \mathrm{~g}$ and $25 \mathrm{~g}$, were purchased from the Laboratory Animal Center, Guangzhou, China, and housed for at least 1 week before use. Tumor cells $\left(3 \times 10^{7}\right.$ cells $\left./ \mathrm{mL}\right)$ were resuspended in PBS 
chilled to $4^{\circ} \mathrm{C}$. To establish subcutaneous tumor xenograft model, $100 \mu \mathrm{L}$ of a cell suspension containing $3 \times 10^{6}$ cells was subcutaneously injected into the dorsal flank of the mice ( $\mathrm{n}=6$ mice per group). To further evaluate the effect of miRNAs on tumor growth, $100 \mu \mathrm{L}$ of miRNA mimics $(2 \mathrm{mg} / \mathrm{mL})$ were injected intratumorally into mice bearing A549 tumors 7 days after inoculation and were administrated every week for 6 weeks. Tumor growth was measured weekly using calipers. Tumor volumes were determined using $1 / 2$ (length $\times$ width $^{2}$ ).

To generate the lung metastasis model, LC cells $\left(3 \times 10^{7}\right.$ cells $\left./ \mathrm{mL}, 50 \mu \mathrm{L}\right)$ were administered intravenously to mice via tail vein infusion ( $\mathrm{n}=6 \mathrm{mice} / \mathrm{group})$. After 4-6 weeks of growth, fresh tumor tissues were surgically removed from the animals after euthanasia and collected for subsequent experiments. To further assess the role of miRNAs on tumor metastasis, $50 \mu \mathrm{L}$ of miRNA mimics were injected via the tail vein after the establishment of the lung metastasis mouse model (once weekly for 6 weeks). The control group was administered an equal volume of PBS via tail vein injection.

To investigate the cytotoxic effect of CIK cells in vivo, subcutaneous tumor xenograft models were generated as described above and mice with similar tumor sizes were selected ( $n=6 /$ group). Mice from each group were administered $50 \mu \mathrm{L}$ of a suspension containing $5 \times 10^{6} \mathrm{CIK}$ cells by peritumoral injection every 3 days, five injections in total. Tumor diameter was also measured weekly and tumor growth curves were plotted. After euthanasia, tumor nodules were excised and tumor volume was measured. Besides, 1week after CIK cell injection, the mice were anesthetized and peripheral blood was obtained by retroorbital bleeding. Cytokine levels and the proportion of CD3+/CD56 + double-positive cells were assessed by flow cytometry.

\section{Statistical analysis}

Data were analyzed using IBM SPSS V.21.0 software (IBM Corp., Armonk, New York, USA). A two-tailed Student's t-test was used for comparisons between two groups. Statistical significance was determined using one-way analysis of variance (ANOVA) with the post hoc Bonferroni test for multiple comparisons. Correlations between different genes were determined by Pearson correlation analysis. Overall survival (OS) of patients with LCs was assessed by the Kaplan-Meier method and log-rank test. P values $<0.05$ were considered statistically significant. All experiments were independently repeated at least three times.

Additional methods are available in online supplemental materials and methods.

\section{RESULTS}

LINC01140 is significantly upregulated in LC tissues and cells

To uncover the biological function of LINC01140 in LC, LINC01140 expression levels in 79 paired LC and normal tissues were tested. We found that LINC01140 expression was greatly upregulated in both lung adenocarcinoma and squamous cell carcinoma tissues compared with adjacent normal samples (figure 1A). High LINC01140 expression in LC samples was associated with advanced tumor-node-metastasis (TNM) stage and distant metastases (figure 1B,C). LC patients with a higher level of LINC01140 showed a worse OS, suggesting that LINC01140 promotes LC progression (figure 1D). In vitro experiments further indicated that LINC01140 expression was higher in LC cells than in human normal lung epithelial cells (figure 1E). The distribution of LINC01140 was measured by extracting RNA from the nucleus and cytoplasm (figure 1F) and lung adenocarcinoma and lung squamous cell carcinoma cells displayed predominantly cytoplasmic LINC01140, which was further validated by fluorescence in situ hybridization (FISH) assay (figure 1G). Consistently, FISH-targeting LINC01140 revealed that LINC01140 expression was stronger in LC tissues than in non-tumor tissues and was also mostly localized to the cytoplasm (figure 1H,I). Besides, in line with the length (3342 nucleotides) by sequencing (online supplemental figure $\mathrm{S} 1$ ), gel electrophoresis of the qPCR products confirmed that these LINC01140 sequences ranged from 3000 to 5000 nucleotides.

Next, LINC01140-knockdown LC cell lines (lung adenocarcinoma cells A549 and lung squamous carcinoma cells EBC-1) were generated by infection with lentivirus carrying LINC01140 shRNA (sh-LINC01140), of which sh1-LINC01140 and sh2-LINC01140 showing high silencing efficiency were selected for subsequent experiments. LINC01140-knockdown LC cells exhibited a marked reduction in proliferative and colonyforming abilities. Besides, flow cytometric analysis of phospho-histone 3 (PHH3)-positive cells confirmed that the percentage of PHH3-positive cells in LINC01140knockdown cells was significantly lower than that in control cells (online supplemental figure S2a-1). Moreover, LINC01140-knockdown LC cells showed attenuated migratory and invasive capacities as determined by scratch and transwell assays (online supplemental figure $\mathrm{S} 2 \mathrm{~m}-\mathrm{p})$.

To determine the effect of LINC0114 knockdown on tumor cell responses to cisplatin (DDP), cell apoptosis was tested by flow cytometry. We found that the percentage of apoptotic cells was increased in LINC01140-knockdown LC cells, as were the intracellular levels of reactive oxygen species (ROS); however, the effect was not significant for sh1-LINC01140, as compared with the wild-type (WT) cells (online supplemental figure S3). Furthermore, compared with the untreated control cells, DDP treatment $(1 \mu \mathrm{g} / \mathrm{mL}, 24$ hours $)$ significantly increased the proportion of apoptotic LINC01140-knockdown LC cells and contributed to increased ROS levels (online supplemental figure S4). Conversely, LINC01140 overexpression significantly enhanced proliferative, migratory, and invasive abilities of LC cells, but decreased the levels of DDP-induced apoptosis (online supplemental figure S5). These findings suggested that LINC01140 functioned as an oncogene, promoting LG cell proliferation, migration, 
A

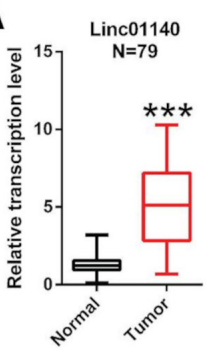

LA+LSCC

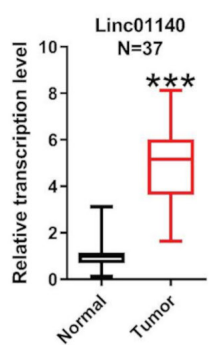

LSCC

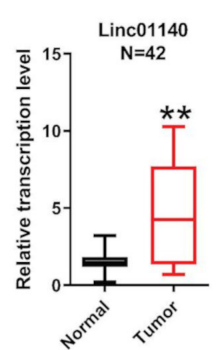

LA
B

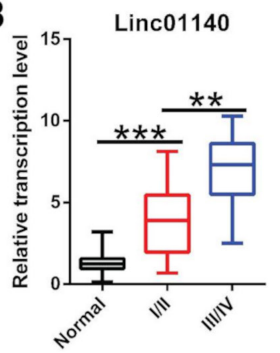

$\mathrm{LA}+\mathrm{LSCC}(\mathrm{N}=79)$

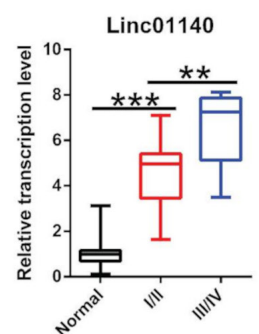

$\operatorname{LSCC}(\mathrm{N}=37)$

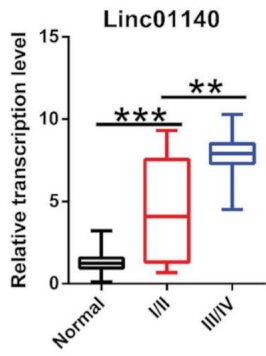

$\mathrm{LA}(\mathrm{N}=42)$

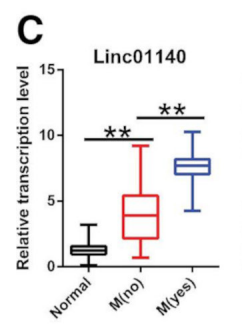

$\mathrm{LA}+\mathrm{LSCC}(\mathrm{N}=79)$

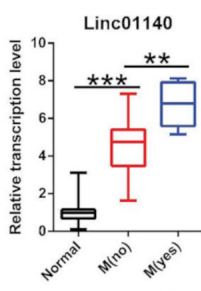

$\operatorname{LSCC}(\mathrm{N}=37)$

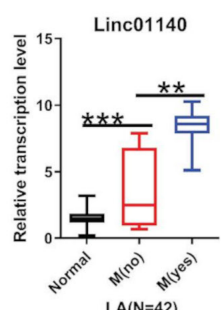

D
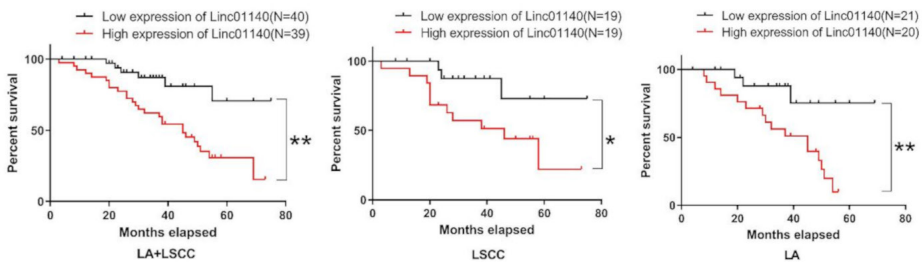

LSCC

E

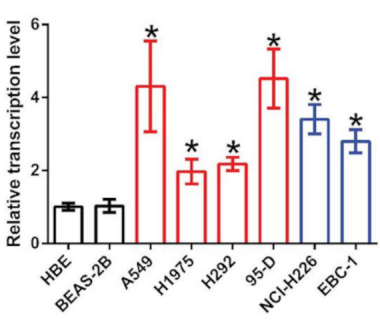

F
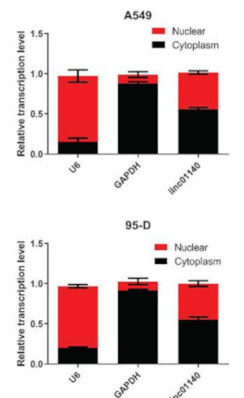

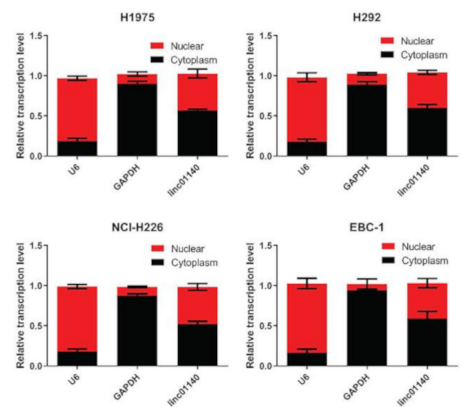

G

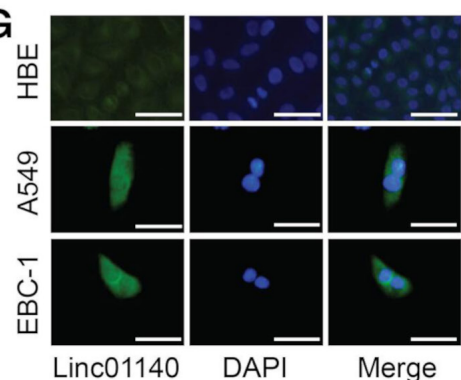

I
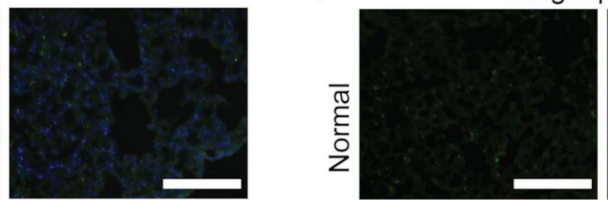

Lung squamous cell carcinoma
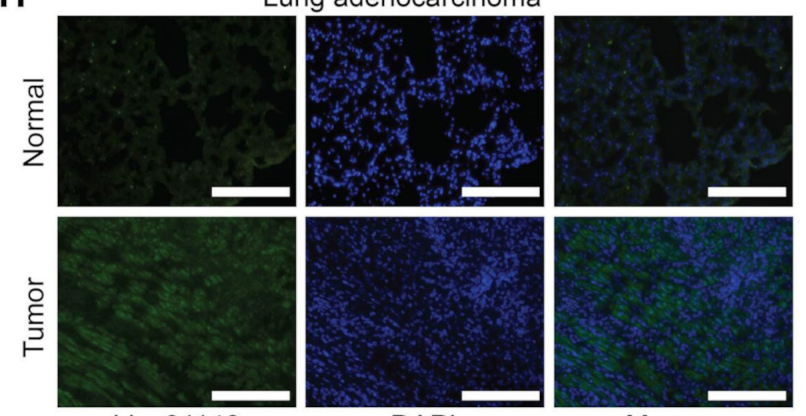

Linc01140

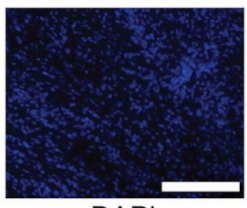

DAPI

Merge

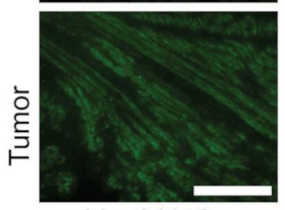

Linc01140

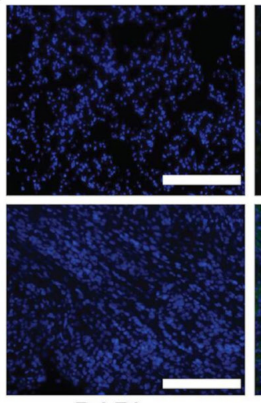

DAPI

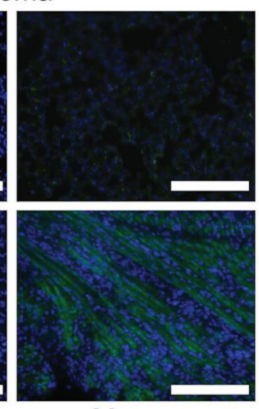

Merge

Figure 1 Long intergenic non-protein coding RNA 1140 (LINC01140) is significantly increased in lung cancer (LC) tissue. (A) QPCR results showed that LINC01140 was significantly upregulated in lung adenocarcinoma and lung squamous cell carcinoma. (B) Stage III/IV lung adenocarcinoma and/or lung squamous cell carcinoma with high expression level of LINC01140 versus stage I/II LC with low expression level of LINC01140. (C) Expression of LINC01140 in tumor tissues of LC patients with metastasis was higher than that in tumor tissues of lung non-metastasis groups. (D) Survival analysis of the LC patients with LINC01140 high and low expression using the Kaplan-Meier survival analysis. High expression of LINC01140 was associated with poor survival outcome in patients with LC. (E) Expression of LINC01140 in LC cells was significantly higher than that in normal lung epithelial cells. (F) Relative LINC01140 expression levels in nuclear and cytosolic fractions of A549, H1975, and H292 cells. Nuclear controls: U6, cytosolic controls: GAPDH. (G) RNA FISH assay revealed LINC01140 mainly located in the cytoplasm of LC cells (A549 and EBC-1) and normal lung epithelial cells HBE. Nuclei was stained by DAPI as blue, LINC01140 sequence was labeled by FAM as green. Scale bar in HBE cells: $50 \mu \mathrm{m}$. Scale bar in A549 and EBC-1 cells: $10 \mu \mathrm{m}$. FISH analysis indicated that LINC01140 was significantly upregulated in $(\mathrm{H})$ lung adenocarcinoma and (I) lung squamous cell carcinoma tissues. Scale bar: $100 \mu \mathrm{m}$. Statistical significance was determined using paired Student's t-test, one-way analysis of variance test, or Kaplan-Meier survival analysis. Error bars, mean \pm SD. Each experiment was repeated at least three times. ${ }^{*} p<0.05$; ${ }^{* *} \mathrm{p}<0.01 ;{ }^{* *} \mathrm{p}<0.001$. FISH, fluorescence in situ hybridization; HBE, human bronchial epithelial; LA, lung adenocarcinoma; LSCC, lung squamous cell carcinoma; GAPDH, glyceraldehyde-3-3phosphate dehydrogenase; DAPI, 4',6-diamidino-2phenylindole; FAM, fluorophore 6-carboxyfluorescein. 
and invasion and suppressing chemotherapy-induced apoptosis in vitro.

\section{LINC01140 promotes the malignant phenotypes of LC cells by acting as miRNAs sponge}

LncRNAs exert 'sponge-like' effects on various miRNAs, thereby inhibiting miRNA-mediated biological functions. Consequently, we assessed potential interactions between LINC01140 and miRNAs. Based on LncBase Predicted v.2 and the LNCediting database, we selected 138 miRNAs scored highly in this analysis. To confirm interactions between LINC01140 and these miRNAs, we performed RNA antisense purification (RAP) using LINC01140 probes and identified interactions for 43 of the 138 miRNAs (online supplemental table S3). Considering the competing endogenous RNA theory, namely, that lncRNA and miRNA expression exhibit opposing trends, we hypothesized that miRNAs with high expression levels in sh1-LINC01140 cells and low expression levels in LINC01140-overexpressing cells were more likely to be directly bound by LINC01140. Of the 43 miRNAs confirmed to interact with LINC01140 (online supplemental figure $\mathrm{S} 6$ ), 25 were significantly downregulated in LINC01140-overexpressing cells (online supplemental table S4), while 18 were markedly upregulated in LINC01140-silenced cells (online supplemental table S5). The intersection of the two sets yielded 13 commonly expressed miRNAs (Venn diagram in figure 2A).

We then performed rescue experiments to further explore which miRNAs were specifically regulated by LINC01140 in LC cells. First, the effects of specific inhibitors (antisense nucleotide strands) of the 13 miRNAs on LC cells were evaluated by cell proliferation, colony formation, and transwell assays. Only miR-33a-5p and miR-33b-5p significantly reversed the LINC01140 knockdown-induced phenotypes (figure 2B-E). Further in vivo experiments demonstrated that LINC01140 knockdown greatly inhibited LC cell growth and metastasis. miR-33a-5p mimics treatment along with LINC01140 knockdown exerted a significant synergistic effect on the proliferative and metastatic capacities of LC cells (figure $2 \mathrm{~F}-\mathrm{H}, \mathrm{J})$. Consistent with the phenotypical findings, immunohistochemistry revealed that LINC01140 knockdown significantly downregulated the expression of proliferation markers Ki-67 and PHH3 in subcutaneous tumors, and treating LINC01140-knockdown cells with miR-33a-5p mimics further decreased immunohistochemistry staining scores of Ki-67 and PHH3 (figure 2I).

\section{LINC01140/miRNAs axis regulates c-Myc expression}

To further confirm that LINC01140 could directly bind to miR-33a-5p and miR-33b-5p and to identify their downstream target genes, the putative binding sites for miR-33a-5p/miR-33b-5p on LINC01140 were predicted by LncBase Predicted v. $2^{21}$ and dual-luciferase reporter assays were performed. c-Myc, a well-known oncogene, was identified as a potential downstream target of miR33a-5p and miR-33b-5p through TargetScanHuman
V.7.2. First, dual-luciferase reporter assays confirmed that mutating the miR-33a-5p and miR-33b-5p binding sites in LINC01140 resulted in a significant decrease in luciferase activity (online supplemental figures S7-S9). Second, a similar result was observed when putative miR-33a-5p and miR-33b-5p binding sites in the c-Myc 3'-untranslated regions (3'-UTR) were mutated. Combined, these findings suggested that both miRNAs directly interacted with LINC01140 and the c-Myc 3'-UTR (figure 3A-D).

Given the reported tumor suppressor roles of miR33a-5p and miR-33b-5p in LC, we then assessed their expression levels in LC tissues. Both miRNAs were significantly downregulated in LC tissues compared with adjacent normal tissues and correlated with TNM stage and distant metastasis (figure 3E-G). The expression levels of c-Myc and LINC01140 were negatively correlated with those of miR-33a-5p and miR-33b-5p, while LINC01140 expression was positively correlated with c-Myc mRNA levels (figure $3 \mathrm{H}-\mathrm{J}$ ). Notably, LC patients with high miR33a-5p and miR-33b-5p expression exhibited longer OS (figure 3K). Besides, miR-33a-5p and miR-33b-5p levels were significantly lower in LC cells than in normal human lung epithelial cells (figure 3L). Compared with controls, the expression of both miRNAs was significantly increased, whereas c-Myc mRNA expression was significantly decreased in LINC01140-knockdown LC cells. Meanwhile, western blotting showed that LINC01140 knockdown significantly reduced c-Myc protein expression, an effect that was partially reversed following miR33a-5p/miR-33b-5p inhibitor treatment (figure 3M-P).

Next, we first confirmed that c-Myc knockdown suppressed the malignant phenotypes of LC cells (online supplemental figure S10). Then, in WT LC cells, treatment with miR-33a-5p or miR-33b-5p inhibitors/mimics significantly downregulated/upregulated miR-33a-5p/ miR-33b-5p expression and increased/decreased c-Myc expression (online supplemental figure S11a,b). Treatment with inhibitors targeting the other 11 abovementioned miRNAs had no effect on c-Myc expression. We further found that c-Myc expression was significantly increased in LINC01140-overexpressing LC cells, but this effect could be rescued by treatment with miR-33a-5p or miR-33b-5p mimics (online supplemental figure S11c,d). These results suggested that LINC01140 promotes LC progression through the miRNAs/c-Myc axis.

\section{miR-377-3p and miR-155-5p, the downstream of LINC01140, directly bind to the $3^{\prime}$-UTR of PD-L1 mRNA, leading to a decrease in PD-L1 expression}

LncRNAs are known to be important in cancer immunity regulation. ${ }^{22}$ PD-L1, also known as $\mathrm{B} 7-\mathrm{H} 1$, is a member of the B7 superfamily that interacts with PD-1 to inhibit T-cell activity and induces effector T-cell apoptosis, thereby impairing antitumor T-cell immunity. ${ }^{23-25}$ As PD-L1 is significantly upregulated in most tumor tissues and represents a highly promising target for immune therapy, we next explored whether the 13 miRNAs predicted to directly bind LINC01140 could affect PD-L1 


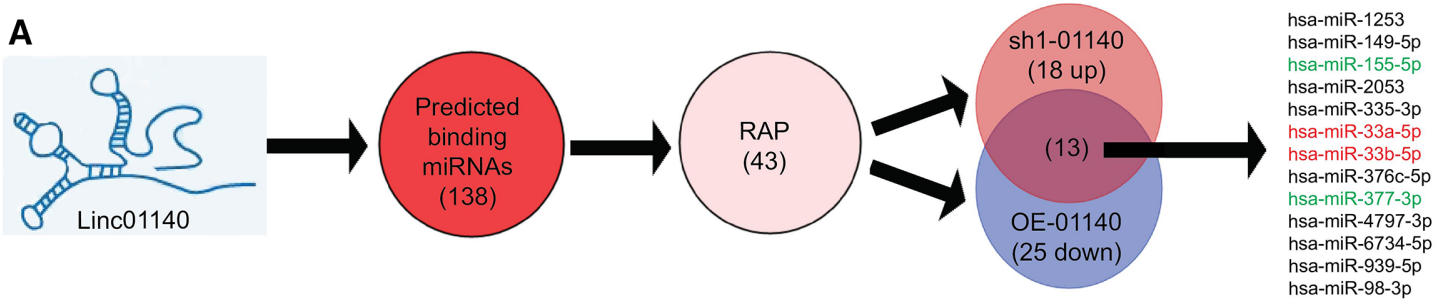

B

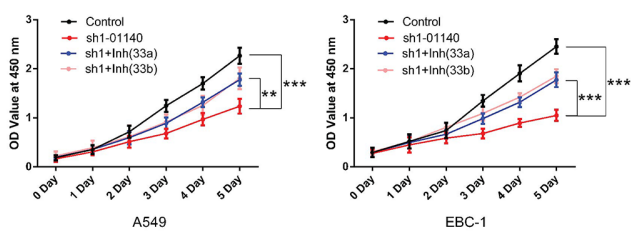

D
C

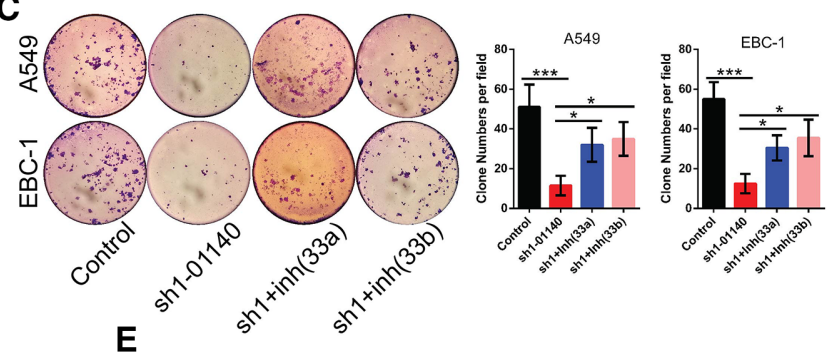

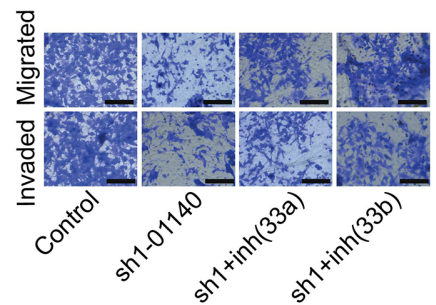

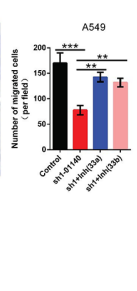

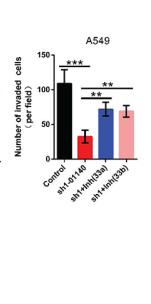

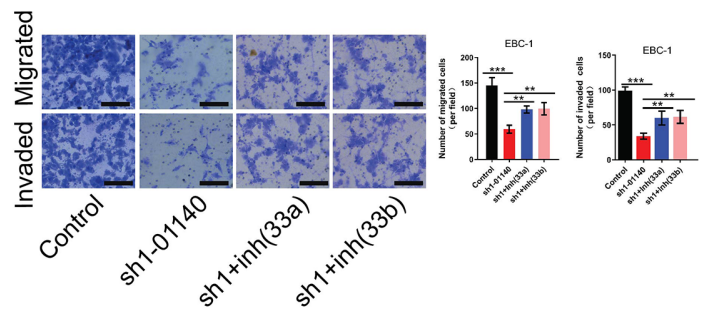

F

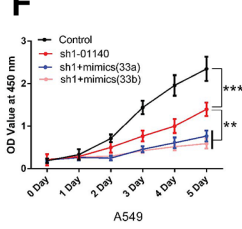

I

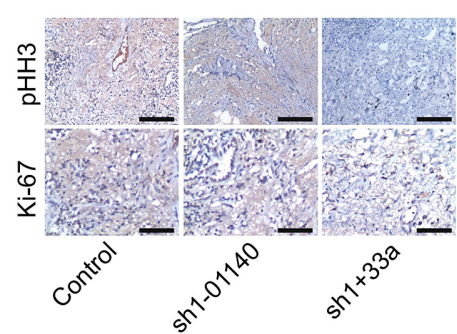

G
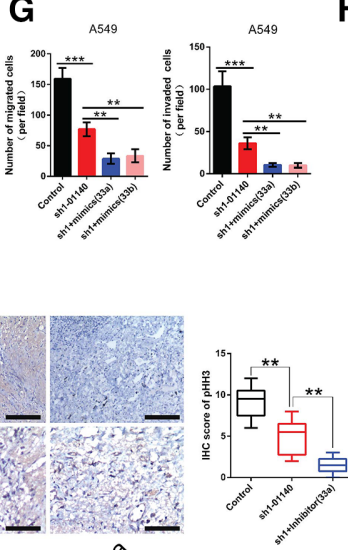

H

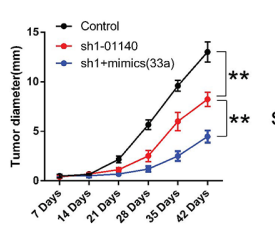

J

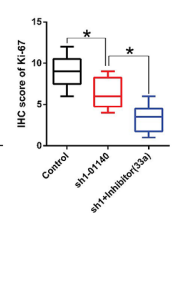

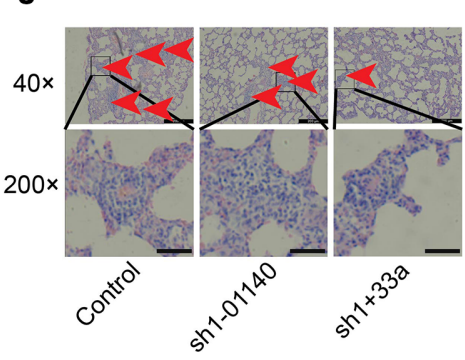
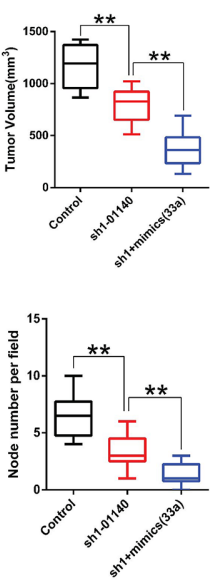

Figure 2 Long intergenic non-protein coding RNA 1140 (LINC01140)/microRNAs (miRNAs) axis modulates the biological behavior of lung cancer (LC) cells. (A) Schematic diagram of the screening strategy to identify miRNAs that directly bound to LINC01140. (B) CCK-8 assay showed that downregulation of miR-33a-5p or miR-33b-5p partially reversed the antiproliferative effects of LINC01140 silencing on LC cells. (C) Colony formation experiments confirmed that miR-33a-5p or miR-33b-5p inhibitors partially reversed LINC01140 knockdown-induced colony formation inhibition in LC cells. (D,E) Transwell assays demonstrated that miR-33a-5p or miR-33b-5p inhibitors partially reversed inhibition of cell migration (top panel) and invasion (bottom panel) by LINC01140 knockdown. Scale bar: $100 \mu \mathrm{m}$. (F,G) CCK-8 and colony formation assays showed that LINC01140 knockdown in combination with upregulation of miR-33a-5p or miR-33b-5p had synergistic inhibitory effect on LC cell proliferation. $(\mathrm{H})$ Xenograft mouse model confirmed that LINC01140 silencing inhibited LC cell growth in vivo, and miR-33a$5 p$ or miR-33b-5p mimics could further repress tumor growth. Scale bar: $1 \mathrm{~cm}$. (I) Immunohistochemical experiment revealed that LINC01140 silencing significantly reduced the protein level of PHH3 and Ki-67 in tumor tissues from the xenograft models ( $n=6$ mice per group) and miR-33a-5p or miR-33b-5p mimics further downregulated their expression levels. Scale bar: $100 \mu m$. (J) H\&E staining demonstrated that LINC01140 silencing reduced the number of tumor nodules in lung metastasis models and miR-33a-5p or miR-33b-5p mimics further suppress lung metastasis in nude mice. Scale bar (upper panels): $400 \mu \mathrm{m}$; Scale bar (lower panels): $10 \mu \mathrm{m}$. Statistical significance was determined using one-way analysis of variance test. Error bars, mean \pm SD. Each experiment was repeated at least three times. ${ }^{*} \mathrm{p}<0.05 ;{ }^{* *} \mathrm{p}<0.01 ;{ }^{* * *} \mathrm{p}<0.001$. RAP, RNA antisense purification; sh, short hairpin RNA; CCK-8, cell counting kit-8. 
A

Linc 01140: 5'-GCUAaUgaugCUAGaAaUCUGCAAUGCAa-3' hsa-miR-33a-5p: 3'-GUUACGUUGAUGUUACGUG-5

Linc 01140: 5'-GCUAAUGAUGCUAGAAAUCUGCAAUGCAA-3' hsa-miR-33b-5p: 3'-CGUUACGUUGUCGUUACGUG-5

C hsa-miR-33a-5p: 3'-GUUACGUUGAUGUUACGUG-5' c-Myc 3'UTR: 5'-UCAAAUGCAUGAUCAAAUGCAAC-3' hsa-miR-33b-5p: 3'-CGUUACGUUGUCGUUACGUG-5'

B

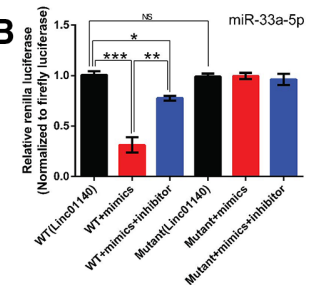

D

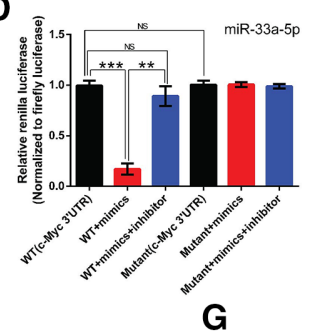

G
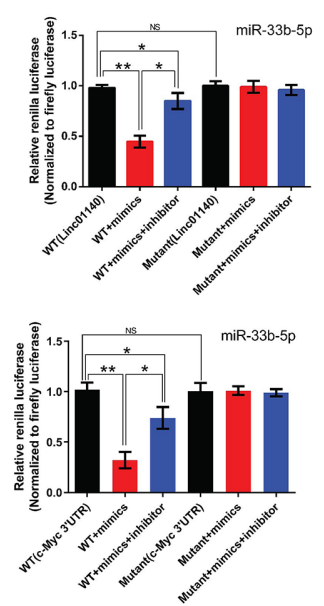

E

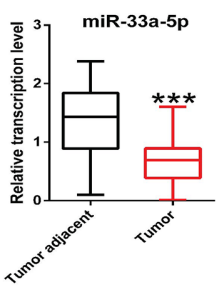

$\mathrm{H}$
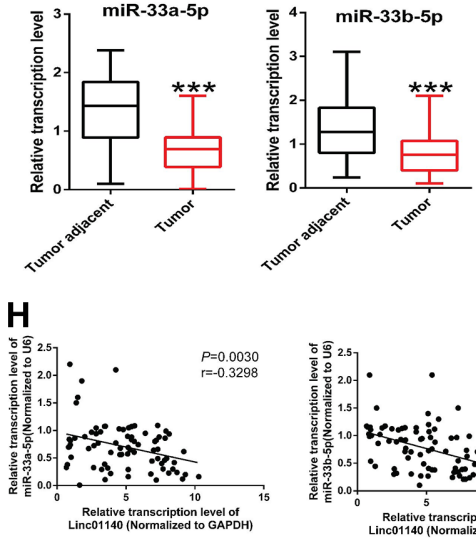

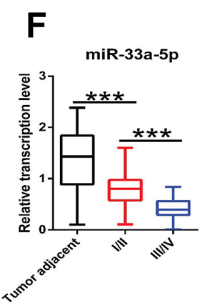

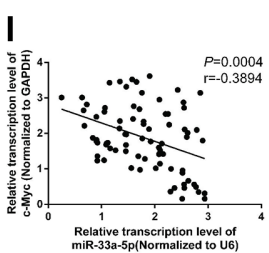

Relative transcription level of
miR-33a-5p(Normalized to U6)

$\mathbf{L}$

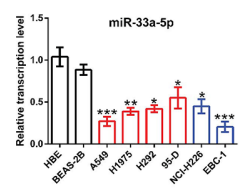

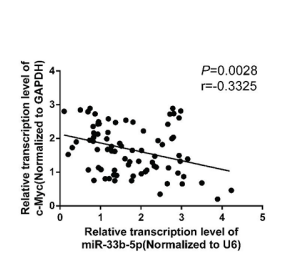

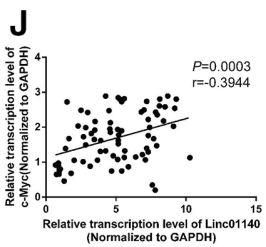

M
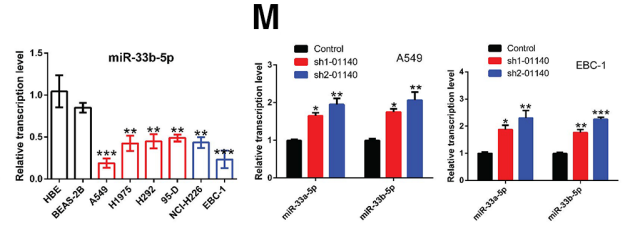
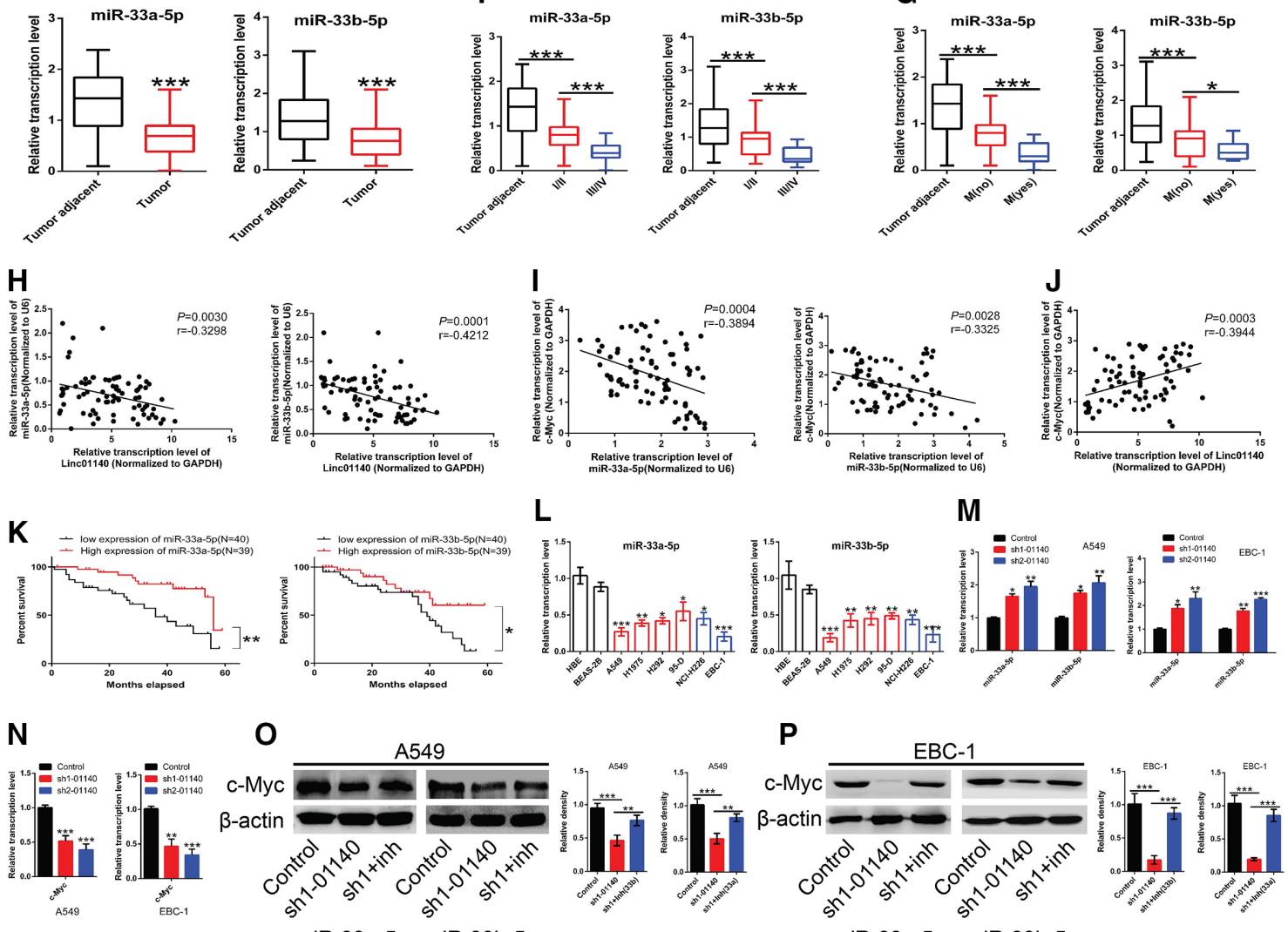

Figure 3 Long intergenic non-protein coding RNA 1140 (LINC01140)/microRNAs (miRNAs) axis modulates c-Myc expression. (A) Schematic representation of the predicted binding sites between LINC01140 and miR-33a-5p (top) or miR-33b-5p (bottom). (B) Luciferase activity in 293T cells transfected with luciferase reporter pmirGLOs containing wild type of LINC01140/mutant construct and miR-3620-3p mimics/inhibitor. (C) Schematic diagram of a predicted binding site of miR-33a-5p (top) or miR33b-5p (bottom) in the $3^{\prime}$-untranslated regions of human c-Myc mRNA. (D) Dual luciferase reporter assays. (E) miR-33a-5p (left) or miR-33b-5p (right) were significantly downregulated in human lung cancer (LC) tissues compared with adjacent non-tumor tissues. (F) MiR-33a-5p or miR-33b-5p expression was highly reduced in stages III-IV LC tissues compared with in stages I-II LC tissues. (G) Expression level of miR-33a-5p or miR-33b-5p in tumor tissues of LC patients with metastasis was significantly lower than that of LC patients without metastasis. (H) LINC01140 expression has a negative correlation with miR-33a-5p (left) or miR-33b-5p (right) expression in LC tissues. (I) The correlation between miR-33a-5p (left) or miR-33b-5p (right) and c-Myc expression in LC tissues. (J) A positive correlation between LINC01140 level and C-Myc expression in LC tissues. (K) Survival analysis revealed that LC patients with high expression of miR-33a-5p or miR-33b-5p had a longer survival time. (L) qPCR analysis of the expression of miR-33a-5p or miR-33b-5p in LC cell lines. (M) qPCR analysis confirmed that miR-33a-5p or miR33b-5p was upregulated in LINC01140-silenced LC cells. (N) LINC01140 knockdown resulted in a significant downregulation of c-MYC mRNA in A549 and EBC-1 cells. (O,P) The protein level of c-MYC. GAPDH and U6 were used as endogenous controls. Statistical significance was determined using paired Student's t-test, one-way analysis of variance test, Pearson correlation analysis, or Kaplan-Meier survival analysis. Error bars, mean \pm SD. Each experiment was repeated at least three times. ${ }^{*} p<0.05$; ${ }^{* *} p<0.01 ;{ }^{* *} p<0.001$. N.S., no significant difference. GAPDH, glyceraldehyde-3-phosphate dehydrogenase. 
A PD-L1 3' UTR: 5'-UCACUUUUUGUACCUGCAUUAAU-3'
hsa-miR-155-5p: 3'-UGGGGAUAGUGCUAAUCGUAII I I I I I PD-L1 3' UTR: 5'-UGUGACAGUGUUCUUUGUGUGAA-3' hsa-miR-377-3p: 3'-UGUUUUCAACGGAAACACACUAA-5'

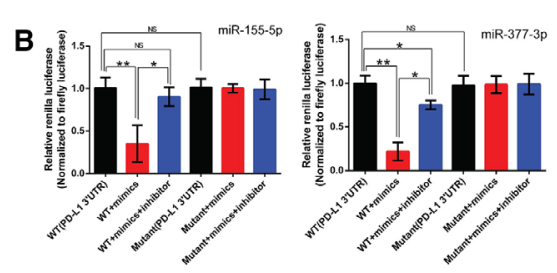

C
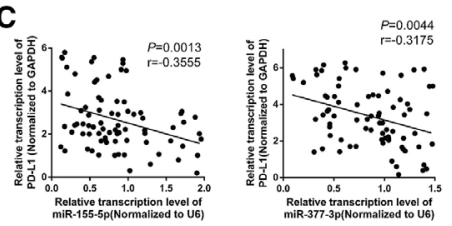
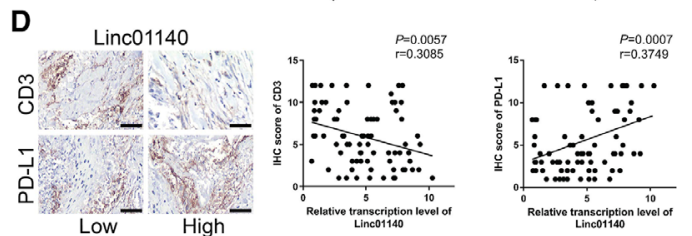

$\mathrm{E}$

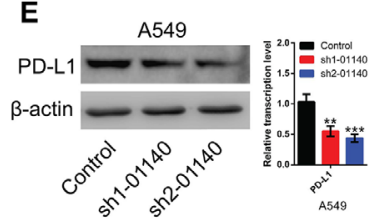

$\mathbf{F}$

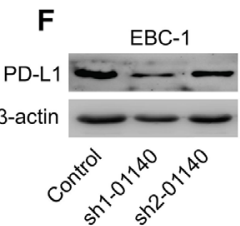

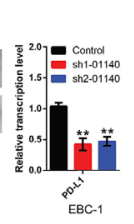

G
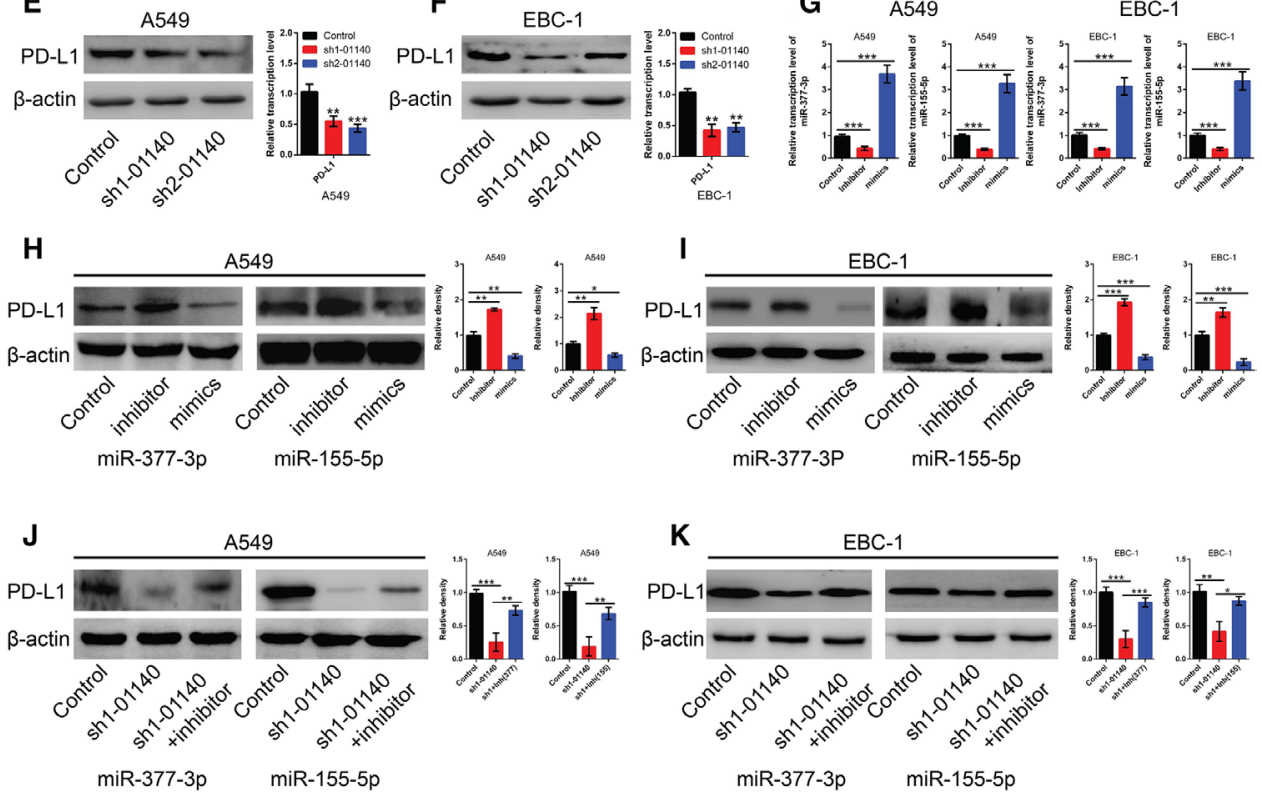

Figure 4 miR-377-3p or miR-155-5 p directly binds to the $3^{\prime}$-untranslated regions ( $3^{\prime}-$ UTR) of PD-L1 mRNA and downregulates its expression. (A) Schematic diagram of the predicted binding sites between the 3'-UTR of PD-L1 mRNA and miR-155-5p (upper panel) or miR-377-3p (lower panel). (B) Dual luciferase reporter assays in 293T cells of miR-377-3p (left) or miR-155-5p (right) target sites predicted in the 3'-UTR of PD-L1. (C) A negative correlation between miR-377-3 p/miR-155-5p and PD-L1 levels in lung cancer (LC) tissues. (D) The expression of long intergenic non-protein coding RNA 1140 (LINC01140) in ILC tissue was positively correlated with PD-L1 IHC score and negatively correlated with CD3 IHC score. Scale bar: $100 \mu \mathrm{m}$. (Silencing of LINC01140 significantly attenuated PD-L1 expression in (E) A549 and (F) EBC-1 cells. (G) MiR-377-3 p/miR-155-5p mimics and the inhibitor significantly increased or decreased the corresponding miRNA level in A549 (left) and EBC-1 (right) cells. MiR-377$3 p$ or miR-155-5 $p$ inhibitor upregulated, while their mimics downregulated the expression of PD-L1 expression in (H) A549 and (I) EBC-1 cells. (J,K) LINC01140 knockdown contributed to the reduction of PD-L1 expression, but miR-377-3p or miR-155$5 p$ inhibitors partially rescued the results caused by knockdown of LINC01140. Statistical significance was determined using one-way analysis of variance test and Pearson correlation test. GAPDH and U6 were used as endogenous controls. Error bars, mean \pm SD. Each experiment was repeated at least three times. ${ }^{*} p<0.05 ;{ }^{* *} p<0.01 ;{ }^{* * *} p<0.001$. N.S., no significant difference. PD-L1, programmed death-ligand 1; IHC, immunohistochemistry; GAPDH, glyceraldehyde-3-phosphate dehydrogenase.

expression. Data showed that treatment with miR-377-3p or miR-155-5 p mimics could dose-dependently reduce PD-L1 expression levels in LC cells.

Next, we predicted the binding sites for miR-377-3p/ miR-155-5p on the 3'-UTR of PD-L1 mRNA through TargetScanHuman V.7.2 (figure 4A). Dual-luciferase reporter assays confirmed that both miR-377-3p and miR-155-5p could bind to the WT PD-L1 3'-UTR sequence, but not when the binding sites were mutated (figure 4B). RAP analysis yielded similar results (online supplemental figure $\mathrm{S} 12 \mathrm{a}, \mathrm{b})$. In the 79 paired LC samples, both miR-377-3 $\mathrm{p}$ and miR-155-5 $\mathrm{p}$ were inversely correlated with PD-L1 mRNA levels (figure 4C). Given that miR-377-3p and miR-155-5 p can bind directly to LINC01140, we then analyzed the correlation between LINC01140 and immune markers in clinical samples. We found that LINC01140 levels were positively correlated with the immunohistochemical score for PD-L1 and CD3 in LC tissues (figure 4D).

LINC01140 knockdown in LC cells significantly downregulated PD-L1 expression relative to controls (figure 4E,F). To elucidate the effect of miR-377-3 $p$ and miR-155-5 p on PD-L1 expression in LC cells, WT LC cells were subjected to treatment with miR-377-3 p/miR-155-5 p 
inhibitors or mimics for 48 hours. qPCR-based validation showed that the levels of both miRNAs were markedly downregulated/upregulated following treatment with the inhibitors/mimics (figure 4G). Western blot results demonstrated that the corresponding inhibitors significantly upregulated PD-L1 expression, whereas the mimics exerted the opposite effects (figure 4H,I). Moreover, treatment with either inhibitor reversed the reduction in PD-L1 expression levels observed in LINC01140knockdown LC cells (figure 4J,K). In contrast, LINC01140 overexpression in LC cells led to a significant increase in PD-L1 expression, which was attenuated by miRNA mimic treatment (online supplemental figure S12c-f).

\section{LINC01140 directly binds to miR-377-3p and miR-155-5p and negatively modulates their expression levels}

To further clarify the LINC01140-mediated effects of miR-377-3 $p$ and miR-155-5 $p$ on PD-L1 expression in LC cells, the putative binding sites for miR-377-3p/ miR-155-5p on LINC01140 were shown in figure 5A and online supplemental figures S13 and S14. Dualluciferase reporter assays confirmed that miR-377-3 $p$ and miR-155-5 p could directly bind to LINC01140 (figure 5B and online supplemental figure S9). RAP assays further confirmed these results (figure 5E). In addition, FISH revealed that LINC01140 colocalized with miR-377-3p and miR-155-5 p in the cytoplasm of LC cells (figure 5C,D) and that miR-377-3 $p$ and miR-155-5 $p$ expression levels were significantly lower in LC cells than in normal human lung epithelial cells (figure 5F). LINC01140 knockdown in LC cells led to a significant increase in miR-377-3 p and miR-155-5 p levels compared with those in the controls (figure 5G).

Further, in LC tissues, miR-377-3p and miR-155-5p levels were significantly decreased and were associated with TNM stage and metastasis (figure $5 \mathrm{H}-\mathrm{J}$ ). Among the 79 patients with LC, those with high miR-377-3p or miR-155-5 p expression had longer OS (figure 5K). Besides, LINC01140 expression level was negatively correlated with miR-377-3 $\mathrm{p}$ and miR-155-5 $\mathrm{p}$ but positively correlated with PD-L1 expression (figure 5L). These findings indicated that the LINC01140/miRNA/PD-L1 axis has a role in the LINC01140-mediated promotion of LC progression.

\section{LINC01140/miRNAs/PD-L1 axis facilitates immune escape in LC}

FISH analysis showed that miR-377-3 $p$ and miR-155-5 $p$ colocalized with PD-L1 mRNA in the cytoplasm of LC cells, providing additional evidence that miR-377-3p and miR-155-5 p regulate PD-L1 expression (figure 6A). Subsequent flow cytometric analysis indicated that LINC01140 knockdown decreased the fluorescence associated with the anti-PD-L1 antibody (left peak); however, miR-377-3 $p$ inhibitor treatment reversed these effects (right peak), indicating that PD-L1 expression on the cell surface could be regulated by LINC01140/miR-377-3p and LINC01140/miR-155-5 $\mathrm{p}$ axis (figure 6B).

To then assess the regulatory role of the LINC01140/ miRNAs/PD-L1 axis on immune responses in LC cells, the cytotoxicity of PBMC-derived CIK cells against LC cells was determined in vitro based on IFN- $\gamma$ production. Phytohemagglutinin (PHA) was used as a control. Coculturing WT LC cells with CIK cells significantly inhibited IFN- $\gamma$ secretion compared with PHA treatment alone. In contrast, IFN- $\gamma$ secretion was significantly elevated in LINC01140-silenced LC cells cocultured with CIK cells at a 1:1 ratio. However, treatment with either the miR-377-3 p or miR-155-5 p inhibitor partially reversed the results induced by LINC01140 knockdown (figure 6C). Data further demonstrated that, when tumor cells were cocultured with CIK cells at different ratios (1:10, 1:20, or 1:40) for 24 hours, cell viability was markedly reduced in LINC01140-knockdown LC cells compared with in WT LC cells, and LDH release levels were significantly higher in LINC01140-knockdown LC cells than in WT LC cells, suggestive of a significant decrease in cellular activity. However, miR-377-3p or miR-155-5 p inhibitor partially reversed the results caused by LINC01140 knockdown in LC cells (figure 6D; online supplemental figure S15). Similar results were observed following bright field images and crystal violet staining of adherent cells (online supplemental figure S16A-D). Conversely, LINC01140 overexpression in LC cells inhibited the capacity of CIK cells to secrete IFN- $\gamma$. MiR-377-3p or miR-377-3p mimics partially rescued the changes induced by LINC01140 overexpression (online supplemental figure S15).

To further evaluate the effect of tumor cells on early apoptosis in CIK cells, we cocultured tumor cells with CIK cells for 24 hours and then quantified the percentage of early apoptotic CIK cells using annexin V-FITC staining. Coculture with LINC01140-knockdown cells significantly reduced the percentage of apoptotic CIK cells compared with coculture with WT cells, while treatment with miR-377-3p mimics further enhanced these effects (online supplemental figure S16e,f). These findings suggested that LINC01140/miRNAs/PD-L1 axis facilitates immune tolerance toward CIK cells.

Next, to confirm the role of the LINC01140/miRNA/ PD-L1 axis on tumor immune escape, we generated subcutaneous xenograft mouse models. When the resulting tumors had grown to approximately the same size, the mice received a peritumoral injection of CIK cells. Compared with the control group, the in vivo growth of LINC01140-knockdown LC cell-derived tumors was significantly suppressed following CIK cell injection. Moreover, treatment with miR-377-3p or miR-155-5p mimics enhanced the cytotoxicity of CIK cells toward LINC01140-knockdown LC cells (figure 6E-H). As miR-377-3P or miR-155-5P mimics did not affect tumor cell proliferation, these effects were likely due to miR-377-3 p or miR-155-5 p-mediated regulation of PD-L1 expression. In support of this possibility, western blotting revealed that LINC01140 knockdown significantly reduced Ki-67, 


\section{A}

Linc 01140: 5'-GCUAAUGCAAUUCCCCAUUAGUGCAUUAA-3'
hsa-miR-155-5p: 3'-UGGGGAUAGUGCUAAUCGUAAUU-5'

Linc 01140: 5'-AUAAUACAUAUAUAAUGAGCAUGUGUGAA-3 hsa-miR-377-3p: 3'-UGUUUUCAACGGAAACACACUA-5'

C

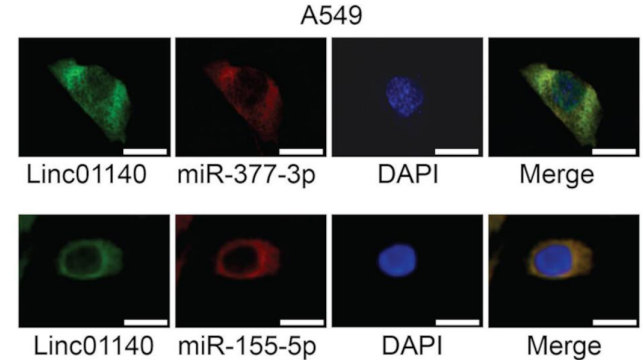

B

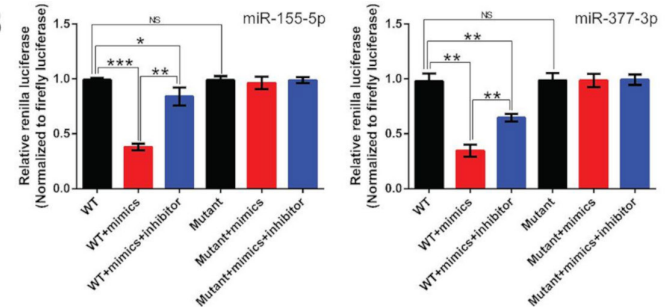

D

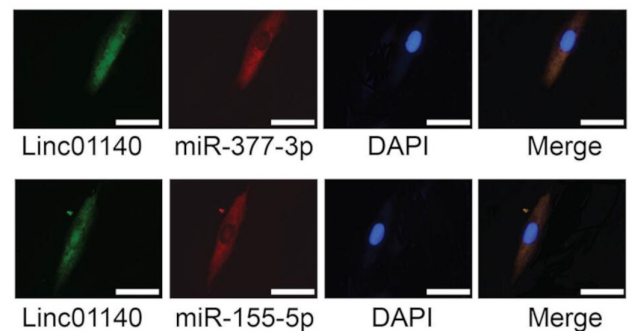

E

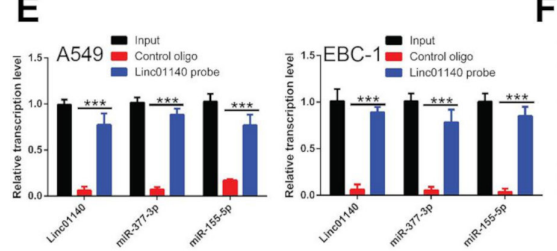

$\mathbf{F}$

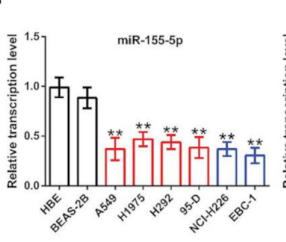

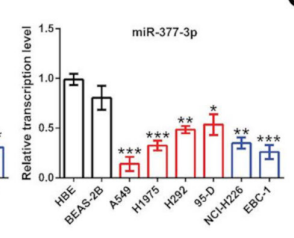

G

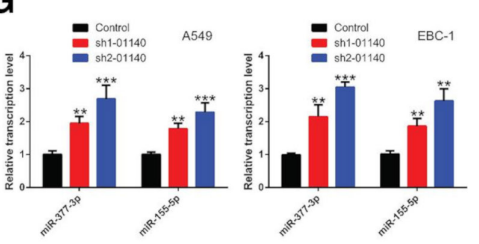

I

$\mathrm{H}$

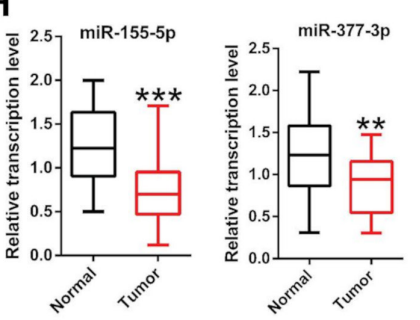

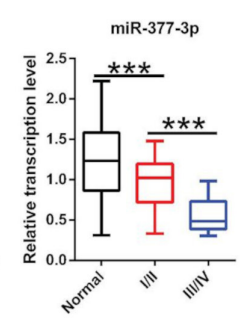
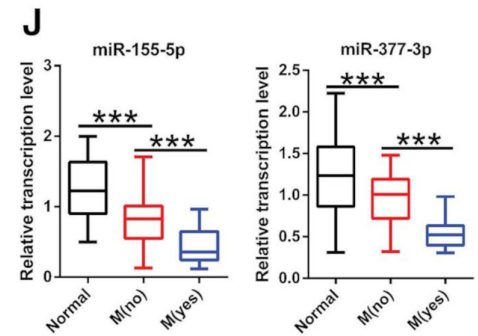
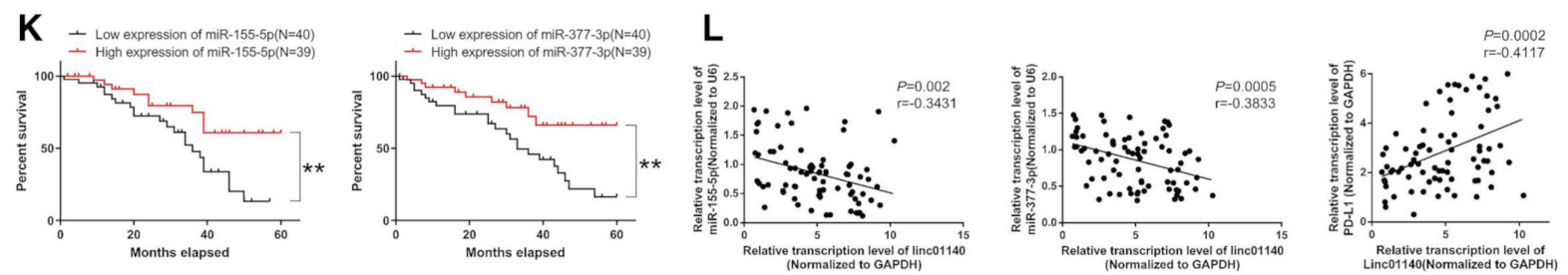

Figure 5 Long intergenic non-protein coding RNA 1140 (LINC01140) directly binds to miR-377-3p and miR-155-5p and negatively regulates their expression. (A) Schematic diagram of putative binding sites between LINC01140 and miR-155-5p (top) or miR-377-3p (bottom). (B) Dual luciferase reporter assays in 293T cells showed the direct interaction between miR-155$5 p$ (left) or miR-377-3p (right) and LINC01140. RNA fluorescence in situ hybridization assay revealed LINC01140 colocalized with miR-155-5p (bottom) or miR-377-3p (top) in the cytoplasm of (C) A549 and (D) EBC-1 cells. Nuclei was stained by DAPI as blue. LINC01140 sequence was labeled by FAM as green. MiR-155-5p or miR-377-3p sequence was labeled by Cy3 as red. Scale bar: $10 \mu \mathrm{m}$. (E) RNA immunoprecipitation experiment in lung cancer (LC) cells confirmed the interaction between LINC01140 and miR-377-3p or miR-155-5 p. (F) Expression levels of miR-377-3p (right) and miR-155-5p (left) in LC cells was significantly lower than in normal human lung epithelial cells. (G) Silencing of LINC01140 in LC cells significantly increased the expression levels of miR-377-3p and miR-155-5p. $(H)$ miR-377-3p and miR-155-5p are significantly downregulated in LC tissues ( $N=79$ ). (I) The expression levels of miR-377-3p and miR-155-5 $p$ in stages III-IV LC are lower than that in stages I-II LC $(\mathrm{N}=79)$. (J) MiR-377-3p and miR-155-5 p levels in the tumor tissues of LC patients with metastasis are lower than that of LC patients without metastasis. (K) LC patients with high expression of miR-377-3p or miR-155-5p had a longer survival time as shown by Kaplan-Meier survival analysis. (L) LINC01140 in LC tissue is negatively correlated with the expression levels of miR$377-3 p$ and miR-155-5p, but positively correlated with the level of PD-L1 mRNA. GAPDH and U6 were used as endogenous controls. Statistical significance was determined using paired Student's t-test, one-way analysis of variance test, Pearson correlation analysis, or Kaplan-Meier survival analysis. Error bars, mean \pm SD. Each experiment was repeated at least three times. ${ }^{*} p<0.05 ;{ }^{* *} p<0.01 ;{ }^{* \star *} p<0.001$. N.S., no significant difference. PD-L1, programmed death-ligand 1; DAPI, 4',6-diamidino2-phenylindole; FAM, fluorophore 6-carboxyfluorescein; GAPDH, glyceraldehyde-3-phosphate dehydrogenase. 

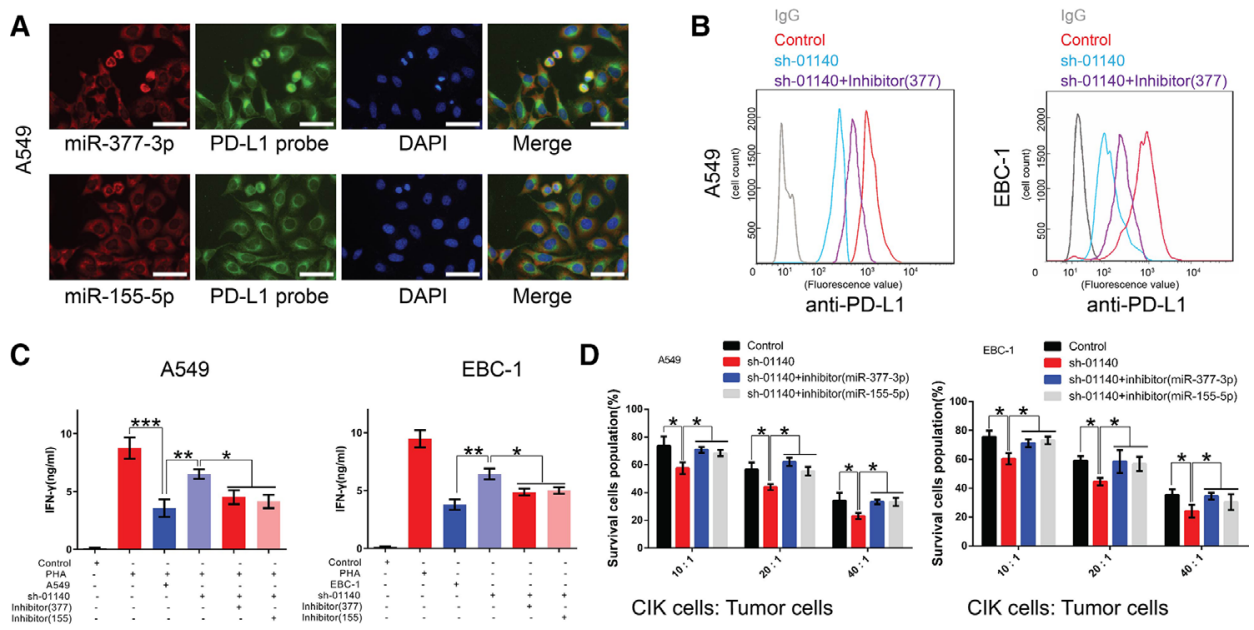

$\mathbf{E}$
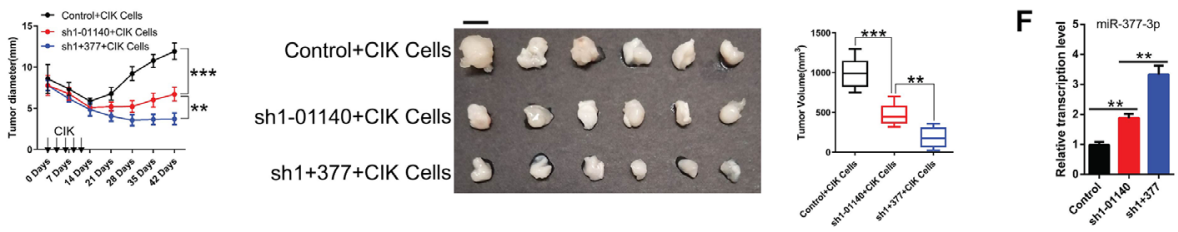

G
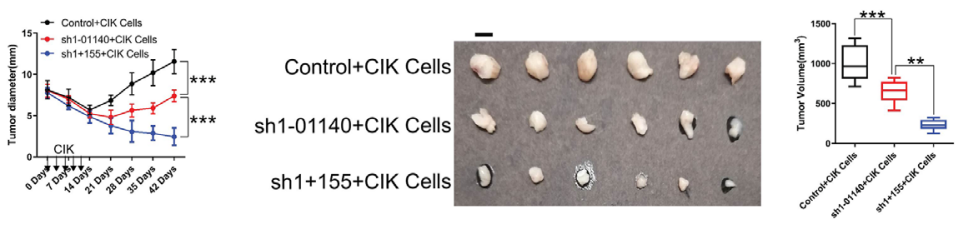

$\mathrm{H}$
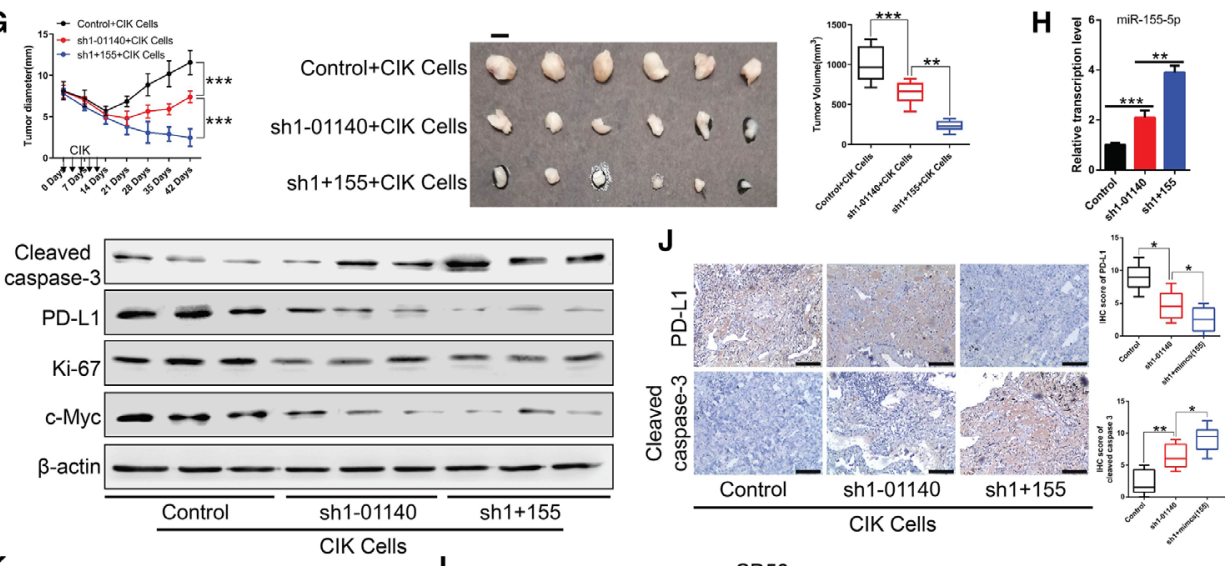

K
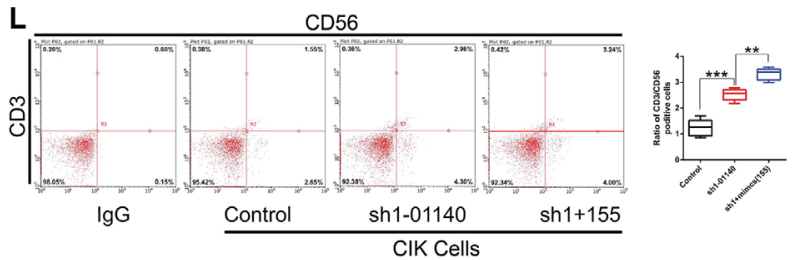

Figure 6 Long intergenic non-protein coding RNA 1140 (LINC01140)/microRNAs/PD-L1 axis mediates immune escape of lung cancer (LC) in vitro and in vivo. (A) RNA fluorescence in situ hybridization experiments confirmed that miR-155$5 p$ (lower panels) or miR-377-3p (upper panels) colocalized with PD-L1 mRNA in the cytoplasm of A549 cells. Scale bar: $50 \mu \mathrm{m}$. (B) Flow cytometry showed that silencing of LINC01140 significantly reduced the expression of PD-L1 in LC cells. (C) Phytohemagglutinin (PHA)-activated human PBMCs were cocultivated with LC cells for 24 hours at $37^{\circ} \mathrm{C}$. And ELISAs confirmed that silencing of LINC01140 elevated the level of IFN- $\gamma$ in the supernatant, but miR-155-5 $p$ or miR-377-3p inhibitors partially reversed the changes caused by LINC01140 knockdown. (D) PHA-activated human PBMCs and LC cells were cocultured at a ratio of 10:1, 20:1, or 40:1, and then CCK-8 was performed to test cell viability. (E) The therapeutic effect of CIK cells on the in vivo growth of tumor cells and representative pictures. Scale bar: $1 \mathrm{~cm}$. (F) miR-377-3p level in xenografted tumor tissues. (G) Tumor growth was monitored prior to, during, and after CIK cell transplantation. (H) miR-155-5p level in xenografted tumor tissues. (I) The protein expression of PD-L1, cleaved caspase-3, Ki-67, and c-Myc in tumor tissues in vivo. (J) Representative images and statistical analysis of IHC staining of PD-L1 and cleaved caspase-3 in xenografted tumors. (K) The levels of interleukin (IL)-2, TNF- $\alpha$, and IFN- $\gamma$ in peripheral blood of tumor-bearing mice were evaluated by ELISA 7 days after CIK cell injection. (L) Representative images and statistical results of $\mathrm{CD} 3^{+} \mathrm{CD} 56^{+}$cells in peripheral blood of tumor-bearing mice determined by flow cytometry 7 days after $\mathrm{CIK}$ cell transplantation. $\mathrm{N}=6$ mice per group. Statistical significance was determined using one-way analysis of variance test. Error bars, mean \pm SD. Each experiment was repeated at least three times. ${ }^{*} p<0.05$; ${ }^{* \star} \mathrm{p}<0.01 ;{ }^{* \star *} \mathrm{p}<0.001$. CIK, cytokine-induced killer. PD-L1, programmed death-ligand 1; DAPI, 4',6-diamidino-2-phenylindole; PBMCs, peripheral blood mononuclear cells; IFN- $\gamma$, interferon-gamma; CCK-8, cell counting kit-8; IHC, immunohistochemistry; TNF- $\alpha$, tumor necrosis factor-alpha. 
c-Myc, and PD-L1 expression, while further treatment with miR-155-5 p mimics downregulated the expression of PD-L1, but not that of Ki-67 or c-Myc (figure 6I). Additionally, LINC01140 knockdown increased the immunohistochemical score for cleaved caspase-3 in xenograft tumor tissues, an effect that was further enhanced by miR-155-5 p mimics treatment (figure 6J), suggesting that CIK cells can induce apoptosis in tumor cells. Importantly, considering that this part of the results was obtained from LC cells with stable knockdown of LINC01140, LINC01140 knockdown caused alterations in cell proliferation and apoptosis as mentioned above. To exclude effects of LINC01140 on tumor progression, we therefore performed experiments using miRNA-377 or miRNA-155 mimics alone following administration with CIK cells in vivo. We found that intratumoral miRNA-377 or miRNA155 mimics injection had no effect on tumor growth compared with control group. However, further CIK cells transfusion significantly restrained tumor growth. It was accompanied with increased cleaved-caspase-3 protein expression and decreased PD-L1 protein expression in tumor tissues. Biological activities of miRNA-377 or miRNA-155 in LC tissues were confirmed to be independent of cell proliferation (online supplemental figure S17). Collectively, these results showed that miRNA-377 or miRNA-155 was able to increase the killing effect of CIK cells by mediating PD-L1 protein expression in comparison to controls.

Further, after CIK cell injection, we measured the cytokine levels in the peripheral blood of mice and found that the levels of IL-2, tumor necrosis factor-alpha (TNF- $\alpha$ ), and IFN- $\gamma$ were significantly increased in mice that had been subcutaneously injected with LINC01140- knockdown LC cells; moreover, the levels of these cytokines were further increased after treatment with miR-377-3p or miR-155-5 p mimics (figure 6K). However, it is not clear whether IFN- $\gamma$ was from tumor cells or CIK cells. Therefore, the validation experiments in vitro were performed. Control cultures were set up with unstimulated PHA and no measurable levels of IFN- $\gamma$ were detected in the culture supernatant of CIK cells. After treatment with stimulating factor PHA, high levels of IFN- $\gamma$ were detected in the CIK cell culture supernatant, indicating that our results were credible. To determine the source of IFN- $\gamma$, we examined supernatant from cultured tumor cells and found that these samples contained low levels of IFN- $\gamma$ but were not significantly different as compared with the blank controls (online supplemental figure S18). We therefore concluded that IFN- $\gamma$ was mainly derived from CIK cells after coculture of CIK cells and tumor cells.

We also measured the proportion of CD3+/CD56 + double-positive cells in the peripheral blood of mice by flow cytometry. The proportion of CD3+/CD56 + double-positive cells was higher in the peripheral blood of mice injected with LINC01140-knockdown LC cells than in that of mice inoculated with control cells and this proportion was further increased following miR-155-5 $\mathrm{p}$ mimic treatment (figure 6L). These findings suggested that CIK cells can penetrate xenografted tissues after tumor cell injection. LINC01140 knockdown suppressed PD-L1 expression, which allowed CIK cells to survive in the tumor tissue for an extended period, resulting in a sustained antitumor effect and the retention of a greater percentage of CIK cells in the peripheral blood of the mice. Overall, these results indicated that the upregulation of LINC01140 expression in LC promotes tumor immune escape through the regulation of the miRNAs/ PD-L1 axis (figure 7).

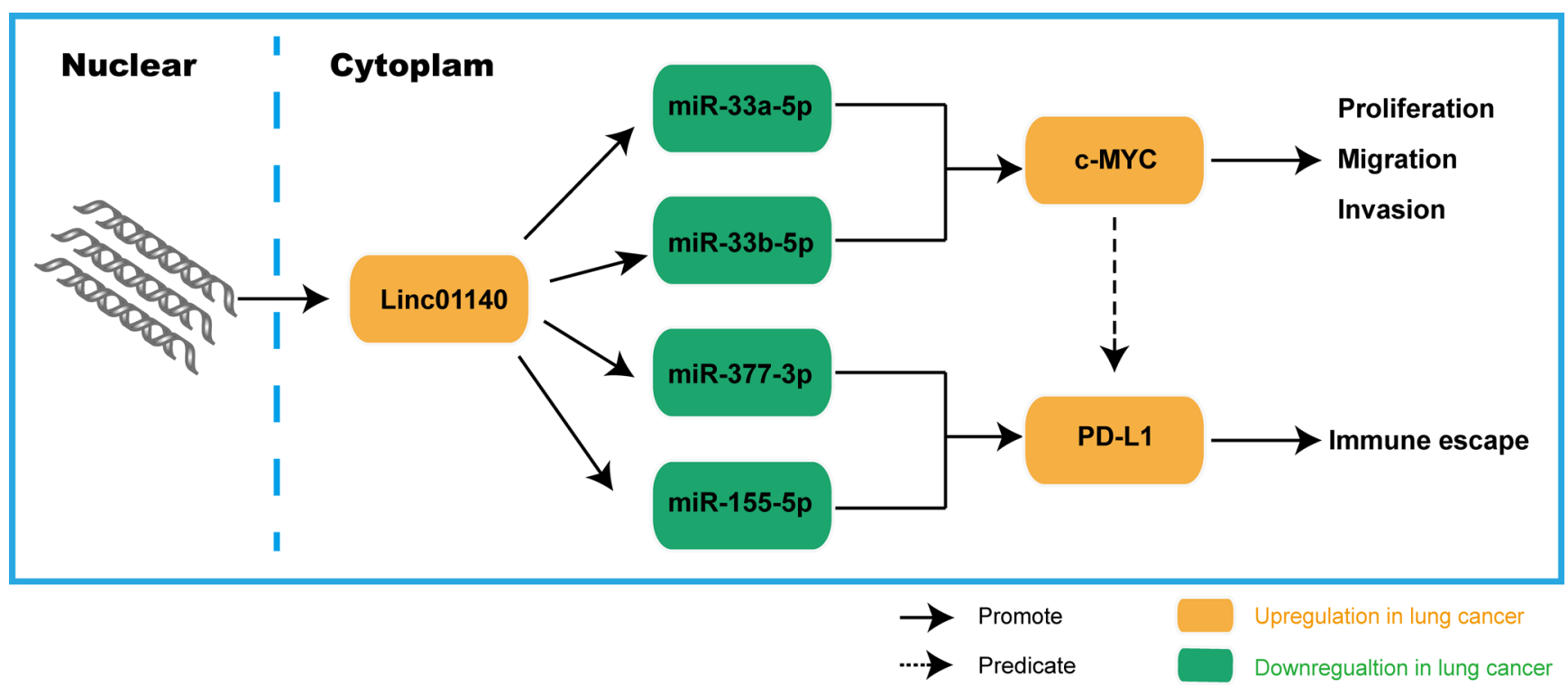

Figure 7 Graphical representation of long intergenic non-protein coding RNA 1140 (LINC01140)-regulated lung cancer (LC) progression and immune escape. Upregulated LINC01140 in LC decreases the expression levels of miR-33a-5p, miR33b-5p, miR-377-3p, and miR-155-5p, resulting in an increase in PD-L1 and c-Myc expression, which finally promotes LC progression and immune escape. PD-L1, programmed death-ligand 1. 


\section{DISCUSSION}

In this study, we observed that the expression of LINC01140 was significantly upregulated in LC tissue and LINC01140 might be a tumor promoter related to LC. Since LC has multiple histological subtypes and many unique clinical and molecular characteristics, ${ }^{26}{ }^{27}$ further exploration of the biological functions of LINC01140, including the molecular features, as well as its relation with drug sensitivity and tumor progression, could assist in guiding the clinical treatment and LC diagnosis. However, recent study has reported that LINC01140 appears to be a tumor suppressor. ${ }^{28}$ Specifically, LINC01140 is downregulated in breast cancer tissues, and on its overexpression, it ultimately affects the epithelial-mesenchymal transition (EMT) process of cells by adsorbing miR-200b and miR-200c, resulting in an antitumor effect. ${ }^{28}$ In addition, another study has found that LINC01140 may inhibit the malignant phenotype of glioma cells by modulating the miR-199a-3p/ZHX1 signaling pathway. ${ }^{29}$ Combining these studies, it is highly suggestive of the tissue specificity of LINC01140, having different effects in different tumors, which provides more possibilities for the research of LINC01140.

LincRNAs have been identified as critical regulators in various cellular processes, including cancer initiation and progression. ${ }^{30}$ Although it has been found that LINC01140 is overexpressed in gastric cancer ${ }^{17}$ and regulates the inflammatory response during pulmonary fibrosis, ${ }^{18}$ the role of LINC01140 in other disease, including LC, is still not completely understood. Recently, an outstanding work by Allou et al revealed that mutations involving lncRNA loci can result in human Mendelian disease. ${ }^{32}$ Therefore, we investigated variants for LINC01140 gene from GeneCards and LincSNP 3.0 databases to enhance our understanding of LINC01140 function and its potential role in cancer. Data showed that 45 single-nucleotide polymorphism (SNP) loci occurred in LINC01140 sequence. And these SNPs in LINC01140 have relationships with various diseases, except for cancer (online supplemental table S6). Certainly, this possibility warrants further exploration.

Since the previous function of LINC01140 was mainly through adsorption of miRNAs, ${ }^{28} 29$ we analyzed the miRNAs that might bind to LINC01140 and screened out 13 miRNAs to which LINC01140 directly binds. With phenotypic rescue experiments, a series of miRNAs that might act as tumor suppressors in LC were discovered, among which miR-33a-5 $p^{33-35}$ and miR-33b-5 $p^{36} 37$ were known as multifunctional miRNAs that were dysregulated in a variety of cancers. Previous studies related to LC suggested that the levels of miR-33a-5 $p^{34}$ and miR-33b-5 $p^{37}$ in plasma and cancer tissue samples were significantly decreased, besides that correlated with the clinicopathological characteristics of the patients significantly. Our study demonstrated that LINC01140 could act as miRNAs 'sponge' to downregulate these miRNAs' intracellular levels by directly adsorbing the miRNAs mentioned above, thus influencing cell proliferation, migration, and invasion. It is a potential mechanism for the downregulation of miR-33a-5p and miR-33b-5p expression in LC.

As LINC01140/miRNAs axis would affect the downstream target genes, we explored the downstream target genes and eventually identified c-Myc as the common target of miR-33a-5p and miR-33b-5p. It mediated the effect of LINC01140/miRNAs axis on LC cell proliferation, migration, and invasion. Notably, it has been proven that c-Myc, a well-known cancer-promoting factor, is significantly upregulated in various cancers, including LC, and is also significantly associated with poor prognosis of patients. ${ }^{38}$ In our study, c-Myc was confirmed to be one of the common target genes of miR-33a-5p and miR-33b-5p, indicating that LINC01140 could promote LC progression by acting on the miRNAs/c-Myc axis.

PD-L1 is a transmembrane protein that is usually upregulated significantly in cancer cells while serving a primary role in suppressing the immune system by binding to PD-1 on the surface of immune cell membranes. ${ }^{23} 40$ There is mounting evidence that upregulated PD-L1 levels are correlated with LC progression and worse prognosis. ${ }^{241}$ Moreover, several preclinical or clinical studies have demonstrated that patients with LC can benefit from anti-PD-L1 antibody therapy. ${ }^{43}$ Considering that tumor cells elevate PD-L1 expression through multiple pathways to evade attack by immune system, we detected a significant reduction in PD-L1 expression levels in cells with LINC01140 non-expressed; meanwhile, the responsiveness of tumor cells to CIK cells appeared to be influenced by LINC01140 knockdown as well. Therefore, we conclude that LINC01140 is capable of regulating PD-L1 expression. After performing rescue experiments with each of the 13 miRNAs previously screened, two miRNAs that possibly affected PD-L1 expression were determined, namely miR-377-3 $\mathrm{p}$ and miR-155-5 p. Earlier studies have shown that miR-377-3 $\mathrm{p}^{4445}$ and miR-155-5 $\mathrm{p}^{4647}$ can act as suppressor factors in a wide range of tumors; however, they have not been shown to regulate PD-L1 expression. Our results provide new insights into the tumor suppressor effects of miR-377-3p and miR-155-5p. Furthermore, this study indicates that LINC01140/miRNAs/PD-L1 axis-mediated signals suppress the expression levels of cytokines (such as IFN- $\gamma$ ) when cocultured with PBMCderived CIK, implying that high expression of LINC01140 may contribute to the escape of tumor cells from immune system attack. These results support the hypothesis that LINC01140-targeted therapy can not only affect the proliferation, migration, and invasion ability of tumor cells by modulating miRNAs but also prevent from tumor immune escape. Interestingly, previous work in various types of human cancer cells suggested that MYC directly binds to the promoter of PD-L1 gene, and MYC inactivation contributed to a rapid downregulation of PD-L1 at the level of transcription or translation. ${ }^{48}$ Therefore, MYC appears to not only promote tumor growth but also drive immune evasion in LC.

Recently, a work in early-stage lung adenocarcinoma revealed that LINC01140 along with other four lncRNAs 
(LINC00857, LINC01116, DRAIC, XIST) can establish novel immune-related lncRNAs signatures for predicting patients' prognosis. ${ }^{49}$ Data from this report strongly support our conclusion that LINC01140 is implicated in tumor immunity. LINC01140 may serve as biomarker for the prognosis of patients with early-stage LUAD and an attractive target for the immunotherapy of LC.

\section{CONCLUSION}

In summary, LINC01140 can be an essential LC-related oncogene that functions to promote LC cell proliferation, migration, invasion, and inhibit apoptosis by restraining antitumor miRNAs. Meanwhile, LINC01140 can also suppress the immune system by upregulating PD-L1 levels in LC cells. These findings provide new perspectives for understanding LC progression, suggesting that LINC01140 may become a prospective novel therapeutic target for LG.

\section{Author affiliations \\ ${ }^{1}$ Department of Medical Oncology, Xiamen Key Laboratory of Antitumor Drug Transformation Research, The First Affiliated Hospital of Xiamen University; School of Clinical Medicine, Fujian Medical University, Xiamen 361003, Fujian Province, China \\ ${ }^{2}$ School of Medicine, Xiamen University, Xiamen 361102, Fujian Province, China ${ }^{3}$ Department of Thoracic Surgery, Xiamen Key Laboratory of Thoracic tumor diagnosis and treatment, Institute of lung cancer, The First Affiliated Hospital of Xiamen University; School of clinical Medicine, Fujian Medical University, Xiamen 361003, Fujian Province, China \\ ${ }^{4}$ Cancer Center, Union Hospital, Tongji Medical College, Huazhong University of Science and Technology, Wuhan 430022, Hubei Province, China}

Acknowledgements The authors thank the reviewers for their valuable comments.

Contributors Guarantor of integrity of the entire study and financial assistance: $X D$, $\mathrm{QL}, \mathrm{JJ}$, and YM. Experimental studies and data analysis: $\mathrm{RX}, \mathrm{GG}, \mathrm{XY}, \mathrm{ZX}, \mathrm{JG}, \mathrm{HL}, \mathrm{NL}$, $\mathrm{ZL}$, and YL. Manuscript preparation: GG, XY, and RX. All authors read and approved the final manuscript.

Funding This study was supported by the National Nature Science Foundation of China (No.81871877), the Natural Science Foundation of Fujian Province (Nos 2016J01636, 2017J01363, 2018J01374), and the Research Fund of Fujian Province Health and Family Planning Commission (Nos 2017-ZQN-86, 2018-CXB-23).

Competing interests None declared.

Patient consent for publication Not required.

Ethics approval All patients were acquired with informed consent, under the protocol approved by the First Affiliated Hospital of Xiamen University Research Ethics Committee.

Provenance and peer review Not commissioned; externally peer reviewed.

Data availability statement All data relevant to the study are included in the article or uploaded as supplementary information.

Supplemental material This content has been supplied by the author(s). It has not been vetted by BMJ Publishing Group Limited (BMJ) and may not have been peer-reviewed. Any opinions or recommendations discussed are solely those of the author(s) and are not endorsed by BMJ. BMJ disclaims all liability and responsibility arising from any reliance placed on the content. Where the content includes any translated material, BMJ does not warrant the accuracy and reliability of the translations (including but not limited to local regulations, clinical guidelines, terminology, drug names and drug dosages), and is not responsible for any error and/or omissions arising from translation and adaptation or otherwise.

Open access This is an open access article distributed in accordance with the Creative Commons Attribution Non Commercial (CC BY-NC 4.0) license, which permits others to distribute, remix, adapt, build upon this work non-commercially, and license their derivative works on different terms, provided the original work is properly cited, appropriate credit is given, any changes made indicated, and the use is non-commercial. See http://creativecommons.org/licenses/by-nc/4.0/.

\section{ORCID iD}

Yanjun Mi http://orcid.org/0000-0002-2796-4657

\section{REFERENCES}

1 Siegel RL, Miller KD, Jemal A. Cancer statistics, 2020. CA Cancer J Clin 2020;70:7-30.

2 Hirsch FR, Scagliotti GV, Mulshine JL, et al. Lung cancer: current therapies and new targeted treatments. Lancet 2017;389:299-311.

3 Barnett R. Lung cancer. Lancet 2017;390:928.

4 Wang X, Adjei AA. Lung cancer and metastasis: new opportunities and challenges. Cancer Metastasis Rev 2015;34:169-71.

5 Popper HH. Progression and metastasis of lung cancer. Cancer Metastasis Rev 2016;35:75-91.

6 Dietrich MF, Gerber DE. Chemotherapy for advanced non-small cell lung cancer. Cancer Treat Res 2016;170:119-49.

7 Kopp F, Mendell JT. Functional classification and experimental dissection of long noncoding RNAs. Cell 2018:172:393-407.

8 Batista PJ, Chang HY. Long noncoding RNAs: cellular address codes in development and disease. Cell 2013;152:1298-307.

9 Kumar MM, Goyal R. Lncrna as a therapeutic target for angiogenesis. Curr Top Med Chem 2017;17:1750-7.

10 Schmitt AM, Chang HY. Long noncoding RNAs in cancer pathways. Cancer Cell 2016;29:452-63.

11 Jarroux J, Morillon A, Pinskaya M. History, discovery, and classification of IncRNAs. Adv Exp Med Biol 2017;1008:1-46.

12 Fatica A, Bozzoni I. Long non-coding RNAs: new players in cell differentiation and development. Nat Rev Genet 2014;15:7-21.

13 Chen L-L. Linking long noncoding RNA localization and function. Trends Biochem Sci 2016;41:761-72.

14 Cui Y, Zhang F, Zhu C, et al. Upregulated IncRNA SNHG1 contributes to progression of non-small cell lung cancer through inhibition of $\mathrm{miR}-101-3 p$ and activation of $\mathrm{Wnt} / \mathrm{\beta}$-catenin signaling pathway. Oncotarget 2017;8:17785-94.

15 Liu W, Yin N-C, Liu H, et al. Cav-1 promote lung cancer cell proliferation and invasion through IncRNA HOTAIR. Gene 2018:641:335-40.

16 Rinn JL, Chang HY. Genome regulation by long noncoding RNAs. Annu Rev Biochem 2012;81:145-66.

17 Song P, Jiang B, Liu Z, et al. A three-IncRNA expression signature associated with the prognosis of gastric cancer patients. Cancer Med 2017;6:1154-64.

18 Hadjicharalambous MR, Roux BT, Csomor E, et al. Long intergenic non-coding RNAs regulate human lung fibroblast function: implications for idiopathic pulmonary fibrosis. Sci Rep 2019;9:6020.

19 Liu Y, Lv J, Liu J, et al. Mucus production stimulated by IFN-AhR signaling triggers hypoxia of COVID-19. Cell Res 2020;30:1078-87.

20 Huang $\mathrm{H}$, Zhu J, Li Y, et al. Upregulation of SQSTM1/p62 contributes to nickel-induced malignant transformation of human bronchial epithelial cells. Autophagy 2016;12:1687-703.

21 Paraskevopoulou MD, Vlachos IS, Karagkouni D, et al. DIANALncBase V2: indexing microRNA targets on non-coding transcripts. Nucleic Acids Res 2016;44:D231-8.

22 Denaro N, Merlano MC, Lo Nigro C. Long noncoding RNAs as regulators of cancer immunity. Mol Oncol 2019;13:61-73.

23 Inoue $\mathrm{Y}$, Yoshimura K, Nishimoto K, et al. Evaluation of programmed death ligand 1 (PD-L1) gene amplification and response to nivolumab monotherapy in non-small cell lung cancer. JAMA Netw Open 2020;3:e2011818.

24 Yu H, Boyle TA, Zhou C, et al. Pd-L1 expression in lung cancer. $J$ Thorac Oncol 2016:11:964-75.

25 Sun C, Mezzadra R, Schumacher TN. Regulation and function of the PD-L1 checkpoint. Immunity 2018;48:434-52.

26 Zheng M. Classification and pathology of lung cancer. Surg Oncol Clin N Am 2016;25:447-68.

27 Zito Marino F, Bianco R, Accardo M, et al. Molecular heterogeneity in lung cancer: from mechanisms of origin to clinical implications. Int $J$ Med Sci 2019;16:981-9.

28 Li D, Li L, Cao Y, et al. Downregulation of LINC01140 is associated with adverse features of breast cancer. Oncol Lett 2020;19:1157-64.

29 Xin Y, Zhang W, Mao C, et al. Lncrna LINC01140 inhibits glioma cell migration and invasion via modulation of miR-199a-3p/ZHX1 axis. Onco Targets Ther 2020;13:1833-44. 
30 Chen W, Yang J, Fang H, et al. Relevance function of Linc-ROR in the pathogenesis of cancer. Front Cell Dev Biol 2020;8:696.

31 Tian B, Han X, Li G, et al. A long intergenic non-coding RNA, LINC01426, promotes cancer progression via AZGP1 and predicts poor prognosis in patients with LUAD. Mol Ther Methods Clin Dev 2020;18:765-80.

32 Allou L, Balzano S, Magg A, et al. Non-Coding deletions identify Maenli IncRNA as a limb-specific En1 regulator. Nature 2021;592:93-8.

33 Wang Y, Zhou X, Shan B, et al. Downregulation of microRNA-33a promotes cyclin-dependent kinase 6 , cyclin D1 and PIM1 expression and gastric cancer cell proliferation. Mol Med Rep 2015:12:6491-500.

34 Pan J, Fang S, Tian $\mathrm{H}$, et al. IncRNA JPX/miR-33a-5p/Twist1 axis regulates tumorigenesis and metastasis of lung cancer by activating Wnt/3-catenin signaling. Mol Cancer 2020;19:9.

35 Jiang N, Wang X, Xie X, et al. IncRNA DANCR promotes tumor progression and cancer stemness features in osteosarcoma by upregulating Axl via miR-33a-5p inhibition. Cancer Lett 2017:405:46-55

36 Huang G, Lai Y, Pan X, et al. Tumor suppressor miR-33b-5p regulates cellular function and acts a prognostic biomarker in RCC. Am J Transl Res 2020;12:3346-60.

37 Zhai S, Zhao L, Lin T, et al. Downregulation of miR-33b promotes non-small cell lung cancer cell growth through reprogramming glucose metabolism miR-33b regulates non-small cell lung cancer cell growth. J Cell Biochem 2019;120:6651-60.

38 O'Donnell KA, Wentzel EA, Zeller KI, et al. C-Myc-Regulated microRNAs modulate E2F1 expression. Nature 2005;435:839-43.

39 Chanvorachote P, Sriratanasak N, Nonpanya N. C-Myc contributes to malignancy of lung cancer: a potential anticancer drug target. Anticancer Res 2020;40:609-18.
40 Tsoukalas N, Kiakou M, Tsapakidis K, et al. Pd-1 and PD-L1 as immunotherapy targets and biomarkers in non-small cell lung cancer. $J$ Buon 2019;24:883-8.

41 Aguilar EJ, Ricciuti B, Gainor JF, et al. Outcomes to first-line pembrolizumab in patients with non-small-cell lung cancer and very high PD-L1 expression. Ann Oncol 2019;30:1653-9.

42 Reck M, Rodríguez-Abreu D, Robinson AG, et al. Pembrolizumab versus chemotherapy for PD-L1-positive non-small-cell lung cancer. N Engl J Med 2016;375:1823-33.

43 Mok TSK, Wu Y-L, Kudaba I, et al. Pembrolizumab versus chemotherapy for previously untreated, PD-L1-expressing, locally advanced or metastatic non-small-cell lung cancer (KEYNOTE-042): a randomised, open-label, controlled, phase 3 trial. Lancet 2019;393:1819-30

44 Huang L, Liu Z, Hu J, et al. MiR-377-3p suppresses colorectal cancer through negative regulation on $\mathrm{Wnt} / \beta$-catenin signaling by targeting XIAP and ZEB2. Pharmacol Res 2020;156:104774.

45 Zhang X-Y, Dong X-M, Wang F-P. MiR-377-3p inhibits cell metastasis and epithelial-mesenchymal transition in cervical carcinoma through targeting SGK3. Eur Rev Med Pharmacol Sci 2020;24:4687-96.

46 Al-Haidari A, Algaber A, Madhi R, et al. MiR-155-5p controls colon cancer cell migration via post-transcriptional regulation of human antigen R (HuR). Cancer Lett 2018;421:145-51.

47 Luo X, Dong J, He X, et al. MiR-155-5p exerts tumor-suppressing functions in Wilms tumor by targeting IGF2 via the PI3K signaling pathway. Biomed Pharmacother 2020;125:109880.

48 Casey SC, Tong L, Li Y, et al. MYC regulates the antitumor immune response through CD47 and PD-L1. Science 2016;352:227-31.

$49 \mathrm{Mu} \mathrm{L}$, Ding K, Tu R, et al. Identification of 4 immune cells and a 5 -IncRNA risk signature with prognosis for early-stage lung adenocarcinoma. J Trans/ Med 2021;19:127. 